\title{
AN ORTHOGONAL MULTIPLE ACCESS CODING SCHEME*
}

\author{
JONATHAN PONNIAH ${ }^{\dagger}$, YIH-CHUN HU ${ }^{\dagger}$, AND P. R. KUMAR
}

\begin{abstract}
Consider a collection of unsynchronized, half-duplex wireless nodes, communicating over a shared spectrum with local affine clocks. The clocks are unsynchronized in the sense that they do not tick at the same rate, called skew, and have different offsets. The nodes also do not know each others rates or offsets. In order for this collection of wireless nodes to even begin to perform any coordinated activity the nodes must first have the fundamental capability to communicate reliably in the presence of half-duplex constraints as well as packet collisions which occur when two or more nodes simultaneous transmit to a common receiver. Both scenarios are also known as primary and secondary conflicts, respectively. We present an orthogonal MAC code that allows any pair in this collection to exchange a message of size $W$ within a bounded time, subject only to the restriction that the ratios of skews have a known upper bound. The orthogonal MAC code described and proved to function in this paper is an essential part of a larger protocol suite. The larger protocol suite addresses the problem of enabling a collection of unsynchronized, half-duplex wireless nodes to also form a fully functioning and indeed optimized wireless network even while under attack from any subset of unknown malicious agents.
\end{abstract}

1. Introduction. Wireless ad-hoc networks have attracted a great deal of research attention over the past decade, in part due to the challenge of operating networks that lack any pre-existing wireless infrastructure. Data transmission in such networks rely on the assistance of intermediate nodes to receive and forward data packets until they arrive at the intended destination. The nodes collectively decide on the routes travelled by the data packets between each source-destination pair, and determine a transmission schedule that supports these routes, all without the assistance of a centralized controller. This distributed real-time operation requires the interaction of many protocols, each of which addresses a unique functional requirement for the network to work reliably. In this paper, we examine one of the most basic requirements; the ability of the nodes to communicate with each other prior to clock synchronization.

The orthogonal MAC protocol that provides the above functionality is an essential

* Dedicated to Professor Hanfu Chen on the occasion of his 75th birthday. This work was supported in part by the National Science Foundation (NSF) under Contracts CCF-0939370, CNS-1035378, CNS-1035340, and by the United States Army Research Oce (USARO) under Contracts W-911NF-0710287 and W911NF-08-1-0238. Any opinions, findings, and conclusions or recommendations expressed in this publication are those of the authors and do not necessarily reflect the views of the above agencies.

$\dagger$ J. Ponniah and Y. Hu are with the Department of Electrical and Computer Engineering, Coordinated Science Laboratory, University of Illinois, Champaign, IL 61801 USA E-mail: \{ponniah1@illinois.edu, yihchun@crhc.uiuc.edu\}

$\ddagger$ P. R. Kumar is with the Department of Electrical and Computer Engineering, Texas A\& M, E-mail: prk@tamu.edu 
component of a larger protocol suite with a more comprehensive goal. Given a set of nodes, some of which are malicious, how can the good nodes form an operating and indeed optimized wireless network? In order to even begin to achieve this larger goal, the nodes must first have the fundamental capability to communicate with each other, even before knowing each others clock skew rates, and do so within a bounded time. The present paper forms part of a collection that together address this overall comprehensive problem.

Wireless transceivers are generally half-duplex; they cannot transmit and receive at the same time due to the physical limitations of the circuitry in their receivers (there is recent work on developing full duplex radios, but endowing a network of only half-duplex nodes to achieve the capability in this paper allows a larger class of networks to operate reliably). Hence, simultaneous transmissions by a transmitter and its intended recipient effectively result in "primary conflicts," a reference to the loss of any messages that arrive while the recipient itself is in "transmit" mode not "receive" mode. A communication scheme that attempts to solve this problem is the orthogonal MAC Gold code [2]. This protocol provides each node with a unique square-pulse waveform, where a pulse defines a time interval in which the node should be in transmit mode. The Gold code has the property that any two waveforms share a non-overlapping pulse during which any transmitted messages will arrive collision-free. However, orthogonal MAC codes are designed under the assumption that the local clocks in the network run at the same speed, even if they are not synchronized. This assumption is not satisfied in practice because distributed clocks are generally subject to skew and offset. Clock skew has the effect of stretching or compressing transmitted signals, a distortion that invalidates the non-overlapping pulse property of the Gold code. The same can be said of any family of waveforms periodic with respect to a single pulse; under compression or stretching the pulses of one waveform can be made to overlap with the pulses of another waveform. In this paper, we solve this problem by designing an orthogonal MAC code based on a family of two-pulse waveforms, in which the spacing between the two pulses in each period is sufficiently asymmetric to ensure that any two linearly distorted waveforms will share a non-overlapping pulse.

A network that lacks a coordinated scheme for communication will likely end up with multiple nodes transmitting messages simultaneously. The above strategy works well to resolve primary conflicts, it does nothing to address a different sort of conflict called "secondary conflict" that occurs when two or more nodes attempt to simultaneously communicate with the same intended recipient. This secondary conflict is also often called a "packet collision." Several protocols have been developed to avoid this from happening in unsynchronized networks. For example ALOHA random access [1], directs a wireless node to back of for an exponentially distributed 
amount of time whenever a collision is detected, and retransmit after this time has expired. However, the probability of repeat collisions within a bounded time-interval, though small, is still nonzero and this uncertainty complicates the design of wireless networks that require guaranteed success.

We overcome the secondary conflict too by dividing the time, as measured by the local clock of each node, into slots, and assigning these slots for communicating to other nodes. A node may only transmit a given message during the time-slot assigned to the corresponding destination. Furthermore, within a time-slot a message can only be transmitted during the windows defined by the orthogonal MAC code pulses. Similarly, any given message must be received during the time-slot assigned to the corresponding source, and outside of the transmission windows defined by the orthogonal MAC code pulses. The orthogonal MAC code presented in this paper ensures that within a bounded time, any source-destination pair will share a nonoverlapping pulse during which the source and destination will be paying attention to each other. We will choose the sizes of the slots, the spacing between the pulses, and the periods of the waveforms, to meet these requirements for any set of clock skews whose ratios are bounded.

The orthogonal MAC code is part of a larger protocol suite [3], (not presented in this paper due to its considerable scope and length) that enables a distributed network of wireless unsynchronized nodes to form a fully functioning wireless network operating at a near optimal rate vector, even under sustained and coordinated attack by malicious nodes hidden amongst them.

The protocol consists of five phases: the neighbor discovery phase, the network discovery phase, the scheduling phase, the data transfer phase, and the verification phase. Good nodes by definition, follow the protocol, and bad nodes attempt to undermine it. Initially, all nodes are powered off, but within a bounded time, all good nodes are guaranteed to have powered on. Each node $i \in\{1, \ldots, n\}$ enters the neighbor discovery phase immediately after start-up, the first move in a broader attempt to obtain a common topological view amongst the good nodes and consistent estimates of the relative clock parameters. Having no knowledge of the topology, node $i$ advertises its presence to the nodes in its neighborhood by broadcasting a probe packet. Between transmissions, node $i$ listens for similar broadcasts and responds to any received probe packet by broadcasting an acknowledgment to the sender. This step, when successfully completed is referred to as a handshake, and it is followed by an exchange of timing packets and mutually authenticated link certificates. The latter contain the relative clock parameters derived from the timing packet measurements.

Two conditions must be satisfied in order for a pair of half-duplex neighbors to complete a handshake: first, a transmitted probe packet must arrive while a neighbor 
is in receive mode; second, an acknowledgement must be returned while the sender of the probe packet is also in receive mode. The chief obstacle to meeting these conditions, is that both nodes are asynchronous; all their attempts at transmission could result in mutual collisions. The orthogonal MAC code we present in this paper is designed to resolve this problem by ensuring that at least one attempt to transmit a message of size $W$ within a fixed time interval will make it through collision-free. As a result, the handshake, the step which precedes all others during the process of forming a network, can be completed within a bounded interval of time. Incidentally, the orthogonal MAC code is used to carry out the remaining steps in the neighbor discovery phase since similar conditions apply to these steps as well. When the neighbor discovery phase is completed, each node is aware of the IDS and relative clock parameters of its neighboring nodes.

The next stage of the protocol occurs during the network discovery phase, in which the nodes disseminate their lists of neighbors amongst themselves and infer a common topological view. However, the behavior of the bad nodes in the network complicates matters. Bad nodes may choose to not cooperate with the protocol, spread false information, or carry out other malicious acts. The protocol uses the Byzantine General's algorithm and a skew consistency test to ensure that the good nodes share the same view of the network topology and have consistent estimates of all the relative clock parameters. Each of these steps are subject to the same conditions as the handshake, and are carried out using the orthogonal MAC code. At the conclusion of the network discovery phase, the nodes are able to schedule their actions based on a sufficiently accurate estimate of a common reference clock. The orthogonal MAC code is no longer needed since the network is able to operate synchronously and schedule collision free transmissions.

Node $i$ then iteratively cycles through the scheduling, data transfer, and verification phases repeatedly, gradually eliminating infeasible concurrent transmission sets until an optimal feasible rate vector is obtained. An important achievement of the protocol is that the rate loss due to overhead, such as the time spent in the neighbor and network discovery phases, can be made arbitrarily small by choosing the duration of each phase appropriately. This result is due to the fact that the length of the orthogonal MAC code is bounded.

The present paper is more focused on the specific problem of uncoordinated communication between half-duplex nodes during the initial stages of network formation, a critical component of the overall protocol suite. In Section 2 we describe the formulation of the orthogonal MAC code in more detail. In Section 3 we prove that the orthogonal MAC code achieves the objectives. In Section 4 we provide an analysis of the efficiency of the orthogonal MAC code. In Section 5 we indicate some direction 


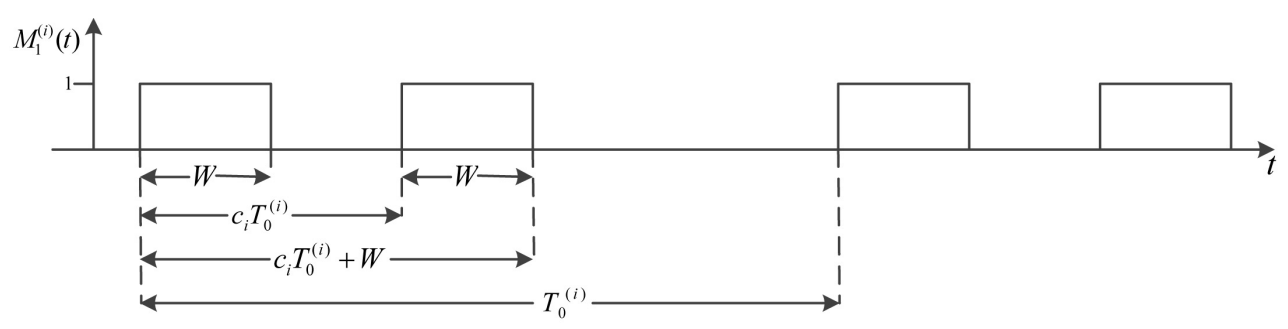

FIG. 1. A graph of $M_{1}^{(i)}(t)$. Note that $M_{2}^{(i)}(t)=M_{1}^{(n-i+1)}(t)$.

for future research.

2. Construction of the Orthogonal MAC code. Consider a collection of $n$ wireless nodes. Each node $i$ is equipped with a local clock $\tau^{i}(t)$ that is affine with respect to some global reference clock $t$. That is, $\tau^{i}(t)=a_{i} t+b_{i}$ where the parameters $a_{i}$ and $b_{i}$ denote the clock skew and offset respectively. Let $a_{i j}>0$ and $b_{i j}$ denote the relative skew and relative offset of node $i$ with respect to node $j$, where $a_{i j}:=\frac{a_{i}}{a_{j}}$ and $b_{i j}:=b_{i}-a_{i j} b_{j}$. Let $\tau_{j}^{i}(t)$ denote node $i$ 's clock with respect to node $j$ 's clock $t$ :

$$
\tau_{j}^{i}(t):=a_{i j} t+b_{i j}
$$

We will assume that $a_{i j} \leq a_{\max }$ for all nodes $i$ and $j$. The orthogonal MAC code for each node $i$ is composed of two fundamental two-pulse waveforms $M_{1}^{(i)}(t)$ and $M_{2}^{(i)}(t)$, which are designed to work when the relative skew expands $\left(a_{i j} \leq\right.$ 1 ) or contracts $\left(a_{i j}>1\right)$, respectively, the transmitted signals received at node $j$. The period of each waveform $M_{1}^{(i)}(t)$ and $M_{2}^{(i)}(t)$ is denoted by $T_{0}^{(i)}$ and $T_{0}^{(n-i+1)}$ respectively, where $T_{0}^{(i)}$ is defined below:

$$
\begin{aligned}
T_{0} & :=32 W n a_{\max }^{2} \\
T_{0}^{(i)} & :=i T_{0}
\end{aligned}
$$

The waveforms $M_{1}^{(i)}(t)$ and $M_{2}^{(i)}(t)$ contain two-pulses of width $W$ that are separated by a distance unique to node $i$. We call the first pulse of the waveform the primary pulse, and the second pulse, the secondary pulse. We use the parameter $c_{i}$ to define the position of the secondary pulse in the waveform, where:

$$
c_{i}:=\frac{1}{a_{\max }(5 n-i)} .
$$




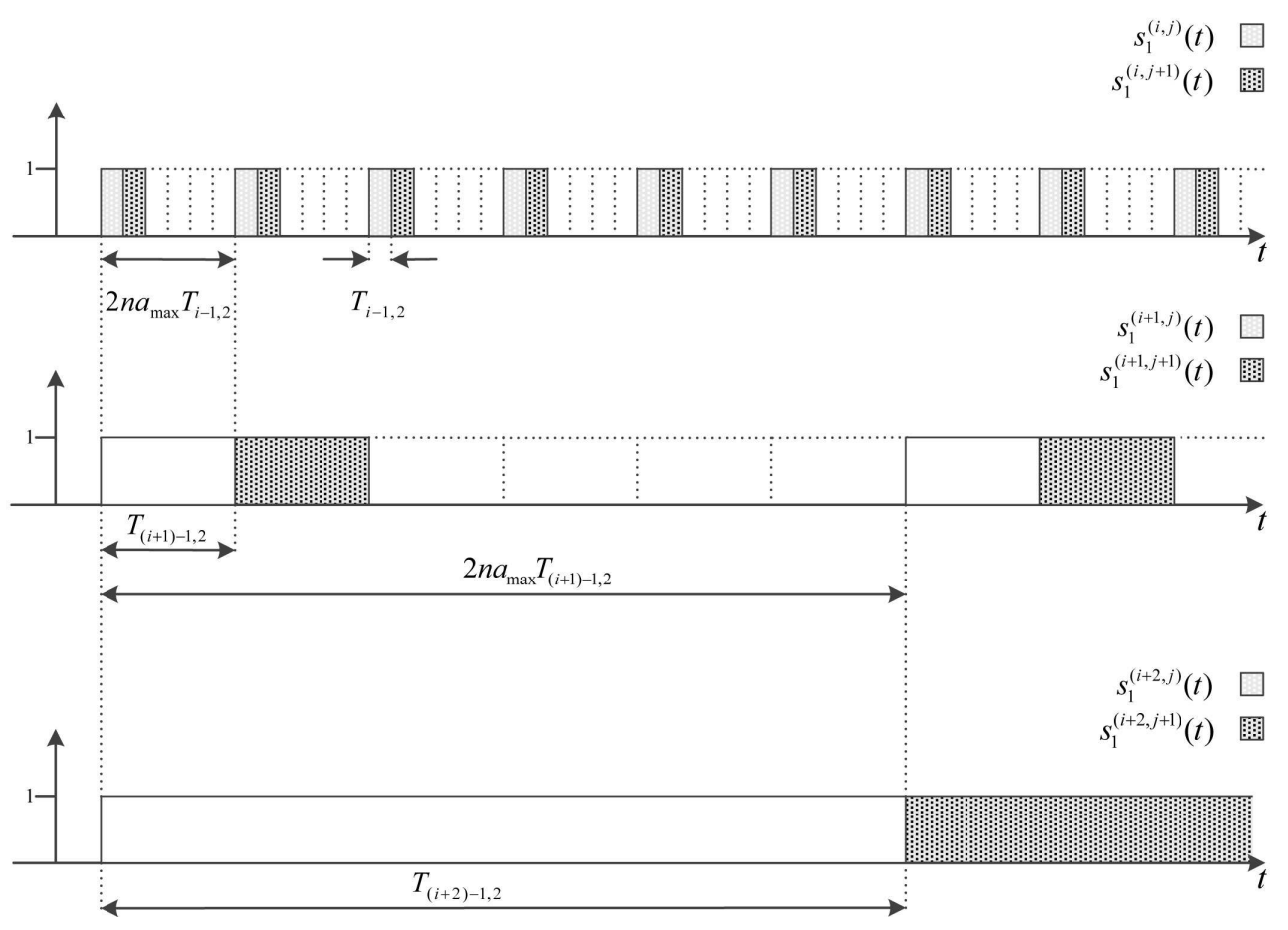

FIG. 2. A graph of $s_{1}^{(i, j)}(t)$. Note that for fixed $i$, the set of functions $\left\{s_{1}^{(i, j)}(t), s_{2}^{(i, j)}(t), j=\right.$ $1, \ldots, n\}$ partition the space $t$. The functions $\left\{s_{1}^{(i+1, j)}(t), s_{2}^{(i+1, j)}(t), j=1, \ldots, n\right\}$ are each constructed to overlap with the set of functions $\left\{s_{1}^{(i, j)}(t), s_{2}^{(i, j)}(t), j=1, \ldots, n\right\}$.

The waveforms $M_{1}^{(i)}(t)$ and $M_{2}^{(i)}(t)$ are defined below (see Figure 1):

$$
\begin{aligned}
& M_{1}^{(i)}(t)= \begin{cases}1 & 0 \leq t \bmod T_{0}^{(i)}<W \\
0 & W \leq t \bmod T_{0}^{(i)}<c_{i} T_{0}^{(i)} \\
1 & c_{i} T_{0}^{(i)} \leq t \bmod T_{0}^{(i)}<c_{i} T_{0}^{(i)}+W \\
0 & c_{i} T_{0}^{(i)}+W \leq t \bmod T_{0}^{(i)}<T_{0}^{(i)}\end{cases} \\
& M_{2}^{(i)}(t)= \begin{cases}1 & 0 \leq t \bmod T_{0}^{(n-i+1)}<W \\
0 & W \leq t \bmod T_{0}^{(n-i+1)}<c_{n-i+1} T_{0}^{(n-i+1)} \\
1 & c_{n-i+1} T_{0}^{(n-i+1)} \leq t \bmod T_{0}^{(n-i+1)} \\
& <c_{n-i+1} T_{0}^{(n-i+1)}+W \\
0 & c_{n-i+1} T_{0}^{(n-i+1)}+W \leq t \bmod T_{0}^{(n-i+1)}<T_{0}^{(n-i+1)} .\end{cases}
\end{aligned}
$$

(3)

In addition, the orthogonal MAC code at each node divides the time as measured by its local clock, into recipient-specific slots assigned for communication by it to every other node. The duration of the time-slots assigned by node $i$ is denoted by 


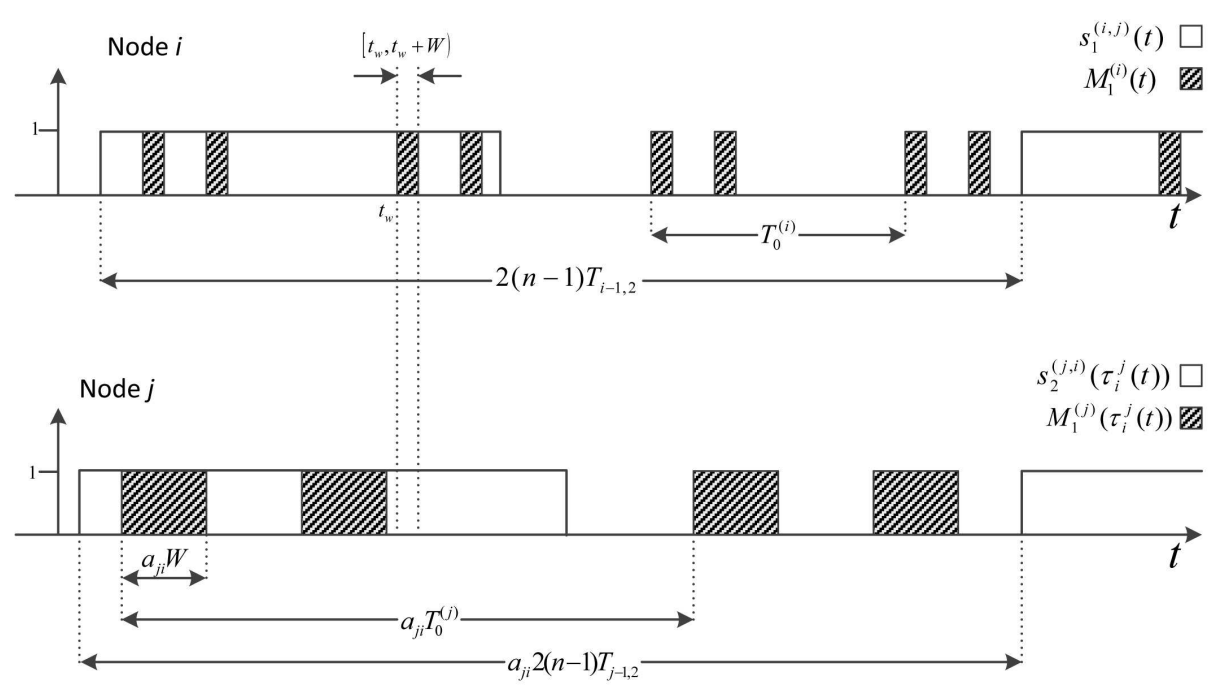

FIG. 3. The interval $\left[t_{w}, t_{w}+W\right)$ satisfies both conditions that are necessary for node $i$ to successfully transmit a message of size $W$ to node $j$. For all $t \in\left[t_{w}, t_{w}+W\right), M_{1}^{(i)}(t) s_{1}^{(i, j)}(t)=1$ and $M_{1}^{(i)}\left(\tau_{i}^{j}(t)\right) s_{1}^{(i, j)}\left(\tau_{i}^{j}(t)\right)+s_{1}^{(i, j)}\left(\tau_{i}^{j}(t)\right)=1$.

$T_{i, 2}$,

$$
\begin{aligned}
T_{0,2} & :=2\left(\left\lceil n a_{\max }\right\rceil+2\right) T_{0}^{(n)}, \\
T_{i, 2} & :=2 a_{\max } n T_{i-1,2} .
\end{aligned}
$$

In each time slot, node $i$ either uses the signal $M_{1}^{(i)}(t)$ or $M_{2}^{(i)}(t)$. We use the functions $s_{1}^{(i, j)}(t)$ and $s_{2}^{(i, j)}(t)$ to define a time-slot associated with the signal $M_{1}^{(i)}(t)$ or $M_{2}^{(i)}(t)$ respectively, that has been assigned by node $i$ to node $j$. The functions $s_{1}^{(i, j)}(t)$ and $s_{2}^{(i, j)}(t)$ are defined below (see Figure 2):

(6)

$$
s_{1}^{(i, j)}(t)=\left\{\begin{array}{rll}
1 & (j-2) T_{i-1,2} & \leq t \bmod 2(n-1) T_{i-1,2} \\
& & <(j-1) T_{i-1,2}, \quad i<j \\
1 & (j-1) T_{i-1,2} & \leq t \bmod 2(n-1) T_{i-1,2} \\
& & <j T_{i-1,2}, \quad i>j \\
0 \quad \text { else }, &
\end{array}\right.
$$

$$
s_{2}^{(i, j)}(t)=\left\{\begin{array}{cc}
1 & (n-1) T_{i-1,2}+(j-2) T_{i-1,2} \leq t \bmod 2(n-1) T_{i-1,2} \\
& <(n-1) T_{i-1,2}+(j-1) T_{i-1,2}, \quad i<j \\
1 & (n-1) T_{i-1,2}+(j-1) T_{i-1,2} \leq t \bmod 2(n-1) T_{i-1,2} \\
& <(n-1) T_{i-1,2}+j T_{i-1,2}, \quad i>j
\end{array}\right.
$$

Now suppose that node $i$ wishes to transmit a message of size $W$ to node $j$ during the interval $\left[t_{s}, t_{s}+T_{M A C}(W)\right)$, where $T_{M A C}(W):=T_{n, 2}$ and $\tau_{i}^{j}\left(t_{s}\right)>0$. 
To accomplish this task, node $i$ transmits its message during the time intervals of size $W$, where both $s_{k}^{(i, j)}(t)=1$ and $M_{k}^{(i)}(t)=1$. The function $s_{k}^{(i, j)}(t)$, when equal to 1 , indicates that node $i$ is "paying attention" to node $j$. That is, node $i$ is either transmitting or listening to node $j$. The function $M_{k}^{(i)}(t)$, when equal to 1 or 0 , determines whether or not node $i$ is transmitting or listening. Taken together, both functions determine when node $i$ is transmitting to node $j$. Therefore to be precise, node $i$ transmits its message to node $j$ during every interval $\left[t_{1}, t_{1}+W\right) \subset$ $\left[t_{s}, t_{s}+T_{M A C}(W)\right)$ that satisfies one of the following condition for all $t \in\left[t_{1}, t_{1}+W\right)$ and some $k \in\{1,2\}$ :

$$
M_{1}^{(i)}(t) s_{1}^{(i, j)}(t)=1
$$

Suppose that node $j$ wishes to receive this message of size $W$ from node $i$ during the interval $\left[\tau_{i}^{j}\left(t_{s}\right), \tau_{i}^{j}\left(t_{s}\right)+T_{M A C}(W)\right)$, where $T_{M A C}(W):=T_{n, 2}$. Node $j$ listens for this message during the time intervals where $s_{k}^{(j, i)}(t)=1$ and $M_{k}^{(j)}(t)=0$. As before, the function $s_{k}^{(j, i)}(t)$, when equal to 1 , indicates that node $j$ is "paying attention" to node $i$. That is, node $j$ is either transmitting or listening to node $i$. Similarly, the function $M_{k}^{(j)}(t)$, when equal to 1 or 0 , determines whether or not node $j$ is transmitting or listening. Taken together, both functions determine when node $j$ is listening to node $i$. Therefore to be precise, node $j$ listens for the message during every interval $\left[t_{1}, t_{2}\right) \subset\left[\tau_{i}^{j}\left(t_{s}\right), \tau_{i}^{j}\left(t_{s}\right)+T_{M A C}(W)\right)$ that satisfies the following condition for all $t \in\left[t_{1}, t_{2}\right)$ and some $k \in\{1,2\}$ :

$$
M_{1}^{(i)}(t) s_{1}^{(i, j)}(t)+s_{1}^{(i, j)}(t)=1 .
$$

The above process is depicted in Figure 3. The following Theorem shows that node $j$ will indeed successfully receive the message.

TheOrem 2.1. Suppose node $i$ transmits a message of size $W$ to node $j$ using the orthogonal MAC code described above. Node $j$ is guaranteed to successfully receive the message transmitted from node $i$. That is, there exists an interval $\left[t_{w}, t_{w}+W\right)$ that satisfies the following conditions for all $t \in\left[t_{w}, t_{w}+W\right)$ and some $k \in\{1,2\}$ :

(iii) $M_{k}^{(i)}(t) s_{k}^{(i, j)}(t)=1$

(iv) $M_{k}^{(j)}\left(\tau_{i}^{j}(t)\right) s_{k}^{(j, i)}\left(\tau_{i}^{j}(t)\right)+s_{k}^{(j, i)}\left(\tau_{i}^{j}(t)\right)=1$

It can be shown that the duration of the orthogonal MAC code satisfies $T_{M A C}(W)$ $\leq c W$ where $c:=\left(2 n a_{\max }\right)^{9 n}$. That is, the duration of the orthogonal MAC code is doubly exponential in the number of nodes. Clearly, this level of efficiency is quite poor. However, as mentioned previously the orthogonal MAC code was designed as part of a larger protocol suite that allows a collection of distributed nodes to form a fully functioning network operating at an optimal rate vector. Since the parameters $n$ and $a_{\max }$ are fixed constants, the effect of the orthogonal MAC code on the protocol 
overhead can be mitigated by choosing a sufficiently long data transfer phase, which in large networks, might require very large time scales. In theory at least, we do not pay a penalty for the relatively poor efficiency of the orthogonal MAC code, but this property will likely require further improvement before the code can be practically implemented.

3. Analysis of the Orthogonal MAC Code Construction. In order to prove that node $j$ can indeed successfully transmit a message to node $i$, we first need to show that there exists an interval of time in which the two nodes are paying attention to each other; the slot allocated to node $i$ by node $j$ overlaps the slot allocated to node $j$ by node $i$. Then, we need to show that somewhere in the intersection of these two time-slots there is a pulse generated by one of the fundamental waveforms $\left\{M_{k}^{(j)}(t), k=1,2\right\}$ that does not collide with a pulse generated by the corresponding waveform $M_{k}^{(i)}\left(\tau_{j}^{i}(t)\right)$ (shown in Figure 3 ). To do this, we locate the first primary pulse generated by $M_{k}^{(j)}(t)$ that occurs in the intersection of the time slots. If this pulse is collision-free, the proof is done. If not, there are two cases to consider. In the first case, the pulse overlaps with a primary pulse generated by $M_{k}^{(i)}\left(\tau_{j}^{i}(t)\right)$. In the second case, the pulse overlaps with a secondary pulse generated by $M_{k}^{(i)}\left(\tau_{j}^{i}(t)\right)$. In each case, we need to show that a non-overlapping pulse generated by node $j$ exists somewhere in the intersection of the two time-slots.

Suppose $i>j$ and $a_{i j} \leq 1$. We use four lemmas to show that node $j$ can transmit a message to node $i$. Lemma 3.1 shows that there exists an interval in which both nodes pay attention to each other. Lemma 3.2 shows that within this interval under case one (mentioned above), there exists a pulse in $M_{1}^{(j)}(t)$ that does not overlap with a pulse in $M_{1}^{(i)}(t)$. Lemma 3.3 shows the same result for case two (mentioned above). Lemma 3.4 ties all three lemmas together to show that node $j$ can indeed successfully transmit a message of length $W$ to node $i$.

Now suppose that $i>j$ and $a_{i j}>1$. We can repeat the above process to show, this time, that node $i$ can transmit a message of length $W$ to node $j$ using the signals $M_{2}^{(i)}(t)$ and $M_{2}^{(j)}(t)$. The proof follows by noting that that $n-j+1>n-i+1, a_{j i} \leq 1$, $M_{2}^{(i)}(t):=M_{1}^{(n-i+1)}(t)$ and $M_{2}^{(j)}(t):=M_{1}^{(n-j+1)}(t)$, and applying the previous four lemmas.

Similarly, we can prove that node $i$ is able to transmit a message of length $W$ to node $j$ when $a_{i j} \leq 1$ using the signals $M_{1}^{(i)}(t)$ and $M_{2}^{(j)}(t)$. Then, repeating the same procedure as before, we can prove that node $j$ can transmit a message of length $W$ to node $i$ when $a_{i j}>1$ using the signals $M_{2}^{(i)}(t)$ and $M_{2}^{(j)}(t)$. We thereby obtain Theorem 3.1 that shows that node $j$ can transmit a non-overlapping pulse to node $i$ and Theorem 3.2 that shows that node $i$ can do the same to node $j$. Putting both theorems together gives us the final proof of Theorem 2.1. 
Consider two nodes $i$ and $j$, where $i>j$ and $a_{i j} \leq 1$. In the following lemma we show that for any pair of nodes $i$ and $j$, there exists an interval of time, $\left[t_{j}, t_{j}+T_{j-1,2}\right)$ with respect to node $j$ 's clock, in which node $i$ and $j$ are scheduled to communicate with each other using the signals $M_{1}^{(i)}(t)$ and $M_{1}^{(j)}(t)$ respectively.

LEMmA 3.1. Without loss of generality assume $i>j$. There exists an interval $\left[t_{j}, t_{j}+T_{j-1,2}\right)$ with respect to node $j$ 's clock in which node $i$ and $j$ are scheduled to communicate with each other using the signals $M_{1}^{(i)}(t)$ and $M_{1}^{(j)}(t)$ respectively. That is, given $t_{1}$ such that $\tau_{j}^{i}\left(t_{1}\right) \geq 0$, the interval $\left[t_{j}, t_{j}+T_{j-1,2}\right)$ satisfies the following conditions:

$$
\begin{aligned}
{\left[t_{j}, t_{j}+T_{j-1,2}\right) } & \subset\left[t_{1}, t_{1}+T_{n, 2}\right), \\
\tau_{j}^{i}\left(\left[t_{j}, t_{j}+T_{j-1,2}\right)\right) & \subset\left[\tau_{j}^{i}\left(t_{1}\right), \tau_{j}^{i}\left(t_{1}\right)+T_{n, 2}\right), \\
s_{1}^{(i, j)}\left(\tau_{j}^{i}(t)\right) & =1, \forall t \in\left[t_{j}, t_{j}+T_{j-1,2}\right), \\
s_{1}^{(j, i)}(t) & =1, \forall t \in\left[t_{j}, t_{j}+T_{j-1,2}\right) .
\end{aligned}
$$

Proof. First we show that there exists an interval $\left[t_{i}, t_{i}+T_{i-1,2}\right) \subset\left[\tau_{j}^{i}\left(t_{1}\right), \tau_{j}^{i}\left(t_{1}\right)+\right.$ $T_{n, 2}$ ) with respect to node $i$ 's clock in which node $i$ pays attention to node $j$. That is, $s_{1}^{(i, j)}\left(\tau^{(i, j)}(t)\right)=1$ for all $t \in \tau_{i}^{j}\left(\left[t_{i}, t_{i}+T_{i-1,2}\right)\right)$.

Since $0 \leq \tau_{j}^{i}\left(t_{1}\right) \bmod 2(n-1) T_{i-1,2}<2(n-1) T_{i-1,2}$, there exists some $t_{a}, 0 \leq$ $t_{a}<2(n-1) T_{i-1,2}$ such that $\left(\tau_{j}^{i}\left(t_{1}\right)+t_{a}\right) \bmod 2(n-1) T_{i-1,2}=(j-1) T_{i-1,2}$ if $2 \leq j \leq n$. Let $t_{i}:=\tau_{j}^{i}\left(t_{1}\right)+t_{a}$. It follows from the definition in (6) that node $i$ is paying attention to node $j$ during the interval $\left.\left[t_{i}, t_{i}+T_{i-1,2}\right)\right)$. Moreover, we have:

$$
\begin{aligned}
t_{i}+T_{i-1,2} & =\tau_{j}^{i}\left(t_{1}\right)+t_{a}+T_{i-1,2} \\
& <\tau_{j}^{i}\left(t_{1}\right)+2(n-1) T_{i-1,2}+T_{i-1,2} \\
& <\tau_{j}^{i}\left(t_{1}\right)+2 n T_{i-1,2} \\
& \leq \tau_{j}^{i}\left(t_{1}\right)+T_{i, 2} \\
& \leq \tau_{j}^{i}\left(t_{1}\right)+T_{n, 2},
\end{aligned}
$$

where (14) follows from the fact that $t_{a}<2(n-1) T_{i-1,2}$, and (15) follows from the definition of $T_{i, 2}$. Therefore it follows from (16) that the interval $\left[t_{i}, t_{i}+T_{i-1,2}\right)$ is contained in one complete run of the orthogonal MAC code. That is, $\left[t_{i}, t_{i}+T_{i-1,2}\right) \subset$ $\left[\tau_{j}^{i}\left(t_{1}\right), \tau_{j}^{i}\left(t_{1}\right)+T_{n, 2}\right)$

Next we show that there exists a sub-interval $\left[t_{j}, t_{j}+T_{j-1,2}\right)$, with respect to node $j$ 's clock, of $\left[t_{i}, t_{i}+T_{i-1,2}\right)$, in which node $j$ pays attention to node $i$. That is, $\tau_{j}^{i}\left(\left[t_{j}, t_{j}+T_{j-1,2}\right)\right) \subset\left[t_{i}, t_{i}+T_{i-1,2}\right)$ and $s_{1}^{j, i}(t)=1$ for all $t \in\left[t_{j}, t_{j}+T_{j-1,2}\right)$.

Now since $0 \leq \tau_{i}^{j}\left(t_{i}\right) \bmod 2(n-1) T_{j-1,2}<2(n-1) T_{j-1,2}$, there exists some $t_{b}$, $0 \leq t_{b}<2(n-1) T_{j-1,2}$ such that $\left(\tau_{i}^{j}\left(t_{i}\right)+t_{b}\right) \bmod 2(n-1) T_{j-1,2}=(i-2) T_{j-1,2}$ if 
$2 \leq i \leq n$. Let $t_{j}:=\tau_{i}^{j}\left(t_{i}\right)+t_{b}$. It follows from the definition of (6) that node $j$ pays attention to node $i$ during the interval $\left[t_{j}, t_{j}+T_{j-1,2}\right)$.

Now we that the interval $\left[t_{j}, t_{j}+T_{j-1,2}\right)$ with respect to node $j$ 's clock is indeed a subinterval of $\left[t_{i}, t_{i}+T_{i-1,2}\right)$ with respect to node $i$ 's clock. That is $\tau_{j}^{i}\left(\left[t_{j}, t_{j}+\right.\right.$ $\left.\left.T_{j-1,2}\right)\right) \subset\left[t_{i}, t_{i}+T_{i-1,2}\right)$. First, we show that $\tau_{j}^{i}\left(t_{j}\right) \geq t_{i}$ :

$$
\begin{aligned}
\tau_{j}^{i}\left(t_{j}\right) & =\tau_{j}^{i}\left(\tau_{i}^{j}\left(t_{i}\right)+t_{b}\right) \\
& =t_{i}+a_{i j} t_{b} \\
& \geq t_{i},
\end{aligned}
$$

where (17) follows from the definition of $t_{j}$. Next, we show that $\tau_{j}^{i}\left(t_{j}+T_{j-1,2}\right)<$ $t_{i}+T_{i-1,2}$ :

$$
\begin{aligned}
\tau_{j}^{i}\left(t_{j}+T_{j-1,2}\right) & =\tau_{j}^{i}\left(\tau_{i}^{j}\left(t_{i}\right)+t_{b}+T_{j-1,2}\right) \\
& =t_{i}+a_{i j} t_{b}+a_{i j} T_{j-1,2} \\
& \leq t_{i}+a_{\max } t_{b}+a_{\max } T_{j-1,2} \\
& <t_{i}+a_{\max } 2(n-1) T_{j-1,2}+a_{\max } T_{j-1,2} \\
& =t_{i}+a_{\max } 2 n T_{j-1,2} \\
& =t_{i}+T_{j, 2} \\
& \leq t_{i}+T_{i-1,2}
\end{aligned}
$$

where (19) follows from the definition of $t_{j},(20)$ follows from the fact that $t_{b}<$ $2(n-1) T_{j-1,2}$, and (23) follows from the definition of $T_{j, 2}$. It follows from (18) and (24) that $\tau_{j}^{i}\left(\left[t_{j}, t_{j}+T_{j-1,2}\right)\right) \subset\left[t_{i}, t_{i}+T_{i-1,2}\right)$. It also follows from (16) that $\tau_{j}^{i}\left(\left[t_{j}, t_{j}+T_{j-1,2}\right)\right) \subset\left[\tau_{j}^{i}\left(t_{1}\right), \tau_{j}^{i}\left(t_{1}\right)+T_{n, 2}\right)$. Therefore node $i$ 's clock is positive (in other words, node $i$ has powered on) during the interval $\tau_{j}^{i}\left(\left[t_{j}, t_{j}+T_{j-1,2}\right)\right)$. Now we show that node $j$ 's clock is positive during the interval $\left[t_{j}, t_{j}+T_{j-1,2}\right)$. We show that $t_{j} \geq t_{1}$ :

$$
\begin{aligned}
t_{j} & =\tau_{i}^{j}\left(t_{i}\right)+t_{b} \\
& \geq \tau_{i}^{j}\left(t_{i}\right) \\
& \geq \tau_{i}^{j}\left(\tau_{j}^{i}\left(t_{1}\right)+t_{a}\right) \\
& =t_{1}+\tau_{i}^{j}\left(t_{a}\right) \\
& \geq t_{1}
\end{aligned}
$$

where (25) follows from the definition of $t_{j},(26)$ follows from the fact that $t_{b}>0$, (27) follows from the definition of $t_{i}$, and (28) follows from the fact that $t_{a}>0$. Next 
we show that $t_{j}+T_{j-1,2}<t_{1}+T_{n, 2}$ :

$$
\begin{aligned}
t_{j}+T_{j-1,2} & =\tau_{i}^{j}\left(t_{i}\right)+t_{b}+T_{j-1,2} \\
& =\tau_{i}^{j}\left(\tau_{j}^{i}\left(t_{1}\right)+t_{a}\right)+t_{b}+T_{j-1,2} \\
& =t_{1}+a_{j i} t_{a}+t_{b}+T_{j-1,2} \\
& <t_{1}+a_{j i} 2(n-1) T_{i-1,2}+2(n-1) T_{j-1,2}+T_{j-1,2} \\
& <t_{1}+a_{j i} 2(n-1) T_{i-1,2}+a_{\max } 2 n T_{j-1,2} \\
& <t_{1}+a_{j i} 2(n-1) T_{i-1,2}+T_{j, 2} \\
& <t_{1}+a_{j i} 2(n-1) T_{i-1,2}+T_{i-1,2} \\
& <t_{1}+a_{\max } 2 n T_{i-1,2} \\
& =t_{1}+T_{i, 2} \\
& \leq t_{1}+T_{n, 2}
\end{aligned}
$$

where (29) follows from the definition of $t_{j},(30)$ follows from the definition of $t_{i},(31)$ follows from the fact that $t_{a}<2(n-1) T_{i-1,2}$ and $t_{b}<2(n-1) T_{j-1,2}$, (32) follows from the definition of $T_{j-1,2}$, (33) follows from the fact that $i>j$, and (34) follows from the definition of $T_{i, 2}$.

It follows from (28) and (35) that the interval $\left[t_{j}, t_{j}+T_{j-1,2}\right)$ occurs in one iteration of the orthogonal MAC code. That is, $\left[t_{j}, t_{j}+T_{j-1,2}\right) \subset\left[t_{1}, t_{1}+T_{n, 2}\right)$. Therefore conditions (10)-(13) are satisfied for the interval $\left[t_{j}, t_{j}+T_{j-1,2}\right)$.

Now we will consider separately the two cases mentioned earlier. First, we consider case one in which a primary pulse generated by node $j$ collides with a primary pulse generated by node $i$. We show that there exists a pulse of length $W$ generated by node $j$ that does not collide with node $i$.

Lemma 3.2. Assume $i>j$ and $a_{i j} \leq 1$. Let $I_{2}:=\left[t_{2}, t_{2}+W\right)$ be a primary pulse of $M_{1}^{(j)}(t)$ with respect to node $j$ 's clock and let $I_{3}:=\left[t_{3}, t_{3}+W\right)$ be a primary pulse of $M_{1}^{(i)}(t)$ with respect to node i's clock. Suppose the two pulses overlap. That is, $\left[t_{2}, t_{2}+W\right) \cap \tau_{i}^{j}\left(\left[t_{3}, t_{3}+W\right)\right) \neq \emptyset$. Then there exists a pulse at $t_{w}:=t_{2}+k T_{0}^{(j)}$ with respect to node $j$ 's clock that does not overlap with any pulse of $M_{1}^{(i)}\left(\tau_{j}^{i}(t)\right)$, where $k:=\left\lfloor\frac{1}{a_{i j}}\left(\frac{i}{j}\right)-\frac{W}{T_{0}^{(j)}}-\frac{W}{a_{i j} T_{0}^{(j)}}-c_{j}\right\rfloor$.

Proof. We will prove the following in sequence:

(i) $k \geq 1$,

(ii) $\left[t_{w}, t_{w}+W\right) \subset \tau_{i}^{j}\left(\left[t_{3}+c_{i} T_{0}^{(i)}+W, t_{3}+T_{0}^{(i)}\right)\right)$,

(iii) $M_{1}^{(j)}(t)=1$ and $M_{1}^{(i)}\left(\tau_{j}^{i}(t)\right)=0$ for all $t \in\left[t_{w}, t_{w}+W\right)$.

If the primary pulse of node $j$ at time $t_{2}$ (wrt to node $j$ 's clock) collides (in other words, the pulses overlap in time) with the primary pulse of node $i$, we show that there exists a primary pulse that does not collide with node $i$ at time $t_{w}:=t_{2}+k T_{0}^{(j)}$ with 
respect to node $j$ 's clock, where $k$ is an integer defined above. Clearly our selection of $k$ cannot be zero because otherwise $t_{w}=t_{2}$, which points to the same pulse that caused the collision in the first place. We show that $k$ as defined in the lemma is always strictly positive. (i) We show that the parameters of the orthogonal MAC code, selected at the onset, guarantees that $k$ is strictly positive. Now by definition $T_{0}:=32 W n a_{\max }^{2}$. Therefore, we prove that $k \geq 1$ due to the following series of inequalities:

$$
\begin{aligned}
& T_{0} \geq 8 W n a_{\max }^{2}, \\
\Rightarrow & \frac{1}{4 n a_{\max }} \geq \frac{2 W a_{\max }}{T_{0}}, \\
\Rightarrow & \frac{1}{n a_{i j}}-c_{n} \geq \frac{2 W}{a_{i j} T_{0}}, \\
\Rightarrow & \frac{[(n-j+1)-(n-i+1)]}{j a_{i j}}-c_{j} \geq \frac{2 W}{j a_{i j} T_{0}}, \\
\Rightarrow & \frac{[(n-j+1)-(n-i+1)]}{j a_{i j}}-\frac{W}{j T_{0}}-\frac{W}{j a_{i j} T_{0}}-c_{j} \geq 0, \\
\Rightarrow & \frac{1}{a_{i j}}+\frac{[(n-j+1)-(n-i+1)]}{j a_{i j}}-\frac{W}{j T_{0}}-\frac{W}{j a_{i j} T_{0}}-c_{j} \geq 1, \\
\Rightarrow & \left|\frac{1}{a_{i j}}\left(\frac{i}{j}\right)-\frac{W}{T_{0}^{(j)}}-\frac{W}{a_{i j} T_{0}^{(j)}}-c_{j}\right| \geq 1, \\
\Rightarrow & k \geq 1 .
\end{aligned}
$$

(ii) Now we show that the pulse generated by node $j$ at $t_{w}$ does not overlap with any pulse generated by node $i$. That is,

$$
\left[t_{w}, t_{w}+W\right) \subset \tau_{i}^{j}\left(\left[t_{3}+c_{i} T_{0}^{(i)}+W, t_{3}+T_{0}^{(i)}\right)\right) .
$$

As before, we show that our choice of parameters for the orthogonal MAC code guarantee this property. First we show that our choice of $T_{0}:=32 W n a_{\text {max }}^{2}$ guarantees that $t_{w} \geq \tau_{i}^{j}\left(t_{3}+c_{i} T_{0}^{(i)}+W\right)$ due to the following sequence of inequalities:

$$
\begin{aligned}
& T_{0} \geq 8 W a_{\max }, \\
& \Rightarrow \quad T_{0} \geq \frac{8 W a_{\max }}{2 a_{\max }-1} \\
& \Rightarrow \quad T_{0} \geq \frac{4 W}{1-\frac{2 n}{4 n a_{\max }}}, \\
& \Rightarrow \quad T_{0} \geq \frac{4 W}{1-2 n c_{n}}, \\
& \Rightarrow \quad T_{0} \geq \frac{4 W}{1-i c_{i}-j c_{j}}, \\
& \Rightarrow \quad T_{0} \geq \frac{4 W}{(i-j)-c_{i}(i-j)-j a_{i j}\left(c_{i}+c_{j}\right)}, \\
& \Rightarrow \quad 1-c_{i}+\frac{\left(1-c_{i}\right)(i-j)}{j a_{i j}} \geq 1+c_{j}+\frac{4 W}{j a_{i j} T_{0}}, \\
& \Rightarrow \quad \frac{1-c_{i}}{a_{i j}}+\frac{\left(1-c_{i}\right)(i-j)}{j a_{i j}} \geq 1+c_{j}+\frac{4 W}{j a_{i j} T_{0}}, \\
& \Rightarrow \quad \frac{1-c_{i}}{a_{i j}}+\frac{\left(1-c_{i}\right)(i-j)}{j a_{i j}} \geq 1+c_{j}+\frac{2 W}{j T_{0}}+\frac{2 W}{j a_{i j} T_{0}}, \\
& \Rightarrow \quad \frac{1}{a_{i j}}\left(\frac{i}{j}\right)-\frac{W}{j T_{0}}-\frac{W}{j a_{i j} T_{0}}-c_{j}-1 \geq \frac{W}{j T_{0}}+\frac{c_{i}}{a_{i j}}\left(\frac{i}{j}\right)+\frac{W}{j a_{i j} T_{0}}, \\
& \Rightarrow \quad\left\lfloor\frac{1}{a_{i j}}\left(\frac{i}{j}\right)-\frac{W}{T_{0}^{(j)}}-\frac{W}{a_{i j} T_{0}^{(j)}}-c_{j}\right\rfloor \geq \frac{W}{T_{0}^{(j)}}+\frac{c_{i}}{a_{i j}}\left(\frac{i}{j}\right)+\frac{W}{a_{i j} T_{0}^{(j)}} \text {, } \\
& \Rightarrow \quad k \geq \frac{W}{T_{0}^{(j)}}+\frac{c_{i}}{a_{i j}}\left(\frac{i}{j}\right)+\frac{W}{a_{i j} T_{0}^{(j)}},
\end{aligned}
$$




$$
\begin{aligned}
& \Rightarrow \quad k T_{0}^{(j)} \geq t_{2}+W-t_{2}+\frac{c_{i} T_{0}^{(i)}}{a_{i j}}+\frac{W}{a_{i j}}, \\
& \Rightarrow \quad k T_{0}^{(j)} \geq \tau_{i}^{j}\left(t_{3}\right)-t_{2}+\frac{c_{i} T_{0}^{(i)}}{a_{i j}}+\frac{W}{a_{i j}} \\
& \Rightarrow \quad t_{2}+k T_{0}^{(j)} \geq \tau_{i}^{j}\left(t_{3}\right)+\frac{c_{i} T_{0}^{(i)}}{a_{i j}}+\frac{W}{a_{i j}} \\
& \Rightarrow \quad t_{w} \geq \tau_{i}^{j}\left(t_{3}+c_{i} T_{0}^{(i)}+W\right) .
\end{aligned}
$$

Next we show that the choice of parameter $c_{j}:=\frac{1}{a_{\max }(5 n-j)}$ guarantees that $t_{w}+W<\tau_{i}^{j}\left(t_{3}+T_{0}^{(i)}\right)$ due to the following sequence of inequalities:

$$
\begin{aligned}
& c_{j}>0, \\
\Rightarrow \quad & \frac{1}{a_{i j}}\left(\frac{i}{j}\right)-\frac{W}{j T_{0}}-\frac{W}{j a_{i j} T_{0}}-c_{j}<\frac{1}{a_{i j}}\left(\frac{i}{j}\right)-\frac{W}{j T_{0}}-\frac{W}{j a_{i j} T_{0}}, \\
\Rightarrow \quad & \left.\quad \frac{1}{a_{i j}}\left(\frac{i}{j}\right)-\frac{W}{j T_{0}}-\frac{W}{j a_{i j} T_{0}}-c_{j}\right\rfloor<\frac{1}{a_{i j}}\left(\frac{i}{j}\right)-\frac{W}{j T_{0}}-\frac{W}{j a_{i j} T_{0}}, \\
\Rightarrow \quad & k<\frac{1}{a_{i j}}\left(\frac{T_{0}^{(i)}}{T_{0}^{(j)}}\right)-\frac{W}{T_{0}^{(j)}}-\frac{W}{a_{i j} T_{0}^{(j)}}, \\
\Rightarrow \quad & k T_{0}^{(j)}<\tau_{i}^{j}\left(t_{3}\right)-\tau_{i}^{j}\left(t_{3}\right)-\frac{W}{a_{i j}}+\frac{T_{0}^{(i)}}{a_{i j}}-W, \\
\Rightarrow \quad & k T_{0}^{(j)}<\tau_{i}^{j}\left(t_{3}\right)-\tau_{i}^{j}\left(t_{3}+W\right)+\frac{T^{(i)}}{a_{i j}}-W, \\
\Rightarrow \quad & k T_{0}^{(j)}<\tau_{i}^{j}\left(t_{3}\right)-t_{2}+\frac{T_{0}^{(i)}}{a_{i j}}-W, \\
\Rightarrow & t_{2}+k T_{0}^{(j)}+W<\tau_{i}^{j}\left(t_{3}\right)+\frac{T_{0}^{(i)}}{a_{i j}}, \\
\Rightarrow \quad & t_{w}+W<\tau_{i}^{j}\left(t_{3}+T_{0}^{(i)}\right) .
\end{aligned}
$$

It follows that $\left[t_{w}, t_{w}+W\right) \subset \tau_{i}^{j}\left(\left[t_{3}+c_{i} T_{0}^{(i)}+W, t_{3}+T_{0}^{(i)}\right)\right)$. (iii) Now we use part (ii) to show that node $j$ transmits and node $i$ is silent during the interval $\left[t_{w}, t_{w}+W\right)$ with respect to node $j$ 's clock. Let $t \in\left[t_{w}, t_{w}+W\right)$. From the fact that $k$ is a positive integer and the definitions of $t_{2}, t_{w}$ we have:

$$
\begin{aligned}
0 & =t_{w} \bmod T_{0}^{(j)} \\
& \leq t_{w} \bmod T_{0}^{(j)}+\left(t-t_{w}\right) \bmod T_{0}^{(j)} \\
& =t_{w} \bmod T_{0}^{(j)}+W \\
& =W .
\end{aligned}
$$

Therefore $0 \leq t_{w} \bmod T_{0}^{(j)}+\left(t-t_{w}\right) \bmod T_{0}^{(j)} \leq W$. Since by definition $W<T_{0}^{(j)}$, it follows that:

$$
\begin{aligned}
0 & \leq t_{w} \bmod T_{0}^{(j)}+\left(t-t_{w}\right) \bmod T_{0}^{(j)} \\
& =\left[t_{w} \bmod T_{0}^{(j)}+\left(t-t_{w}\right) \bmod T_{0}^{(j)}\right] \bmod T_{0}^{(j)} \\
& =t \bmod T_{0}^{(j)} \\
& <W .
\end{aligned}
$$

By the inequality $0 \leq t \bmod T_{0}^{(j)}<W$ for all $t \in\left[t_{w}, t_{w}+W\right)$ shown above, and the definition of $M_{1}^{(j)}(t)$, it follows that $M_{1}^{(j)}(t)=1$ for all $t \in\left[t_{2}, t_{2}+W\right)$. So we have 
proven that node $j$ transmits during the interval $\left[t_{w}, t_{w}+W\right)$. Now we need to show that node $i$ is silent.

We have $t \in\left[\tau_{i}^{j}\left(t_{3}+c_{i} T_{0}^{(i)}+W\right), \tau_{i}^{j}\left(t_{3}+T_{0}^{(i)}\right)\right)$ since $\left[t_{2}, t_{2}+W\right) \subset\left[\tau_{i}^{j}\left(t_{3}+c_{i} T_{0}^{(i)}+\right.\right.$ $W), \tau_{i}^{j}\left(t_{3}+T_{0}^{(i))}\right)$. Then $\tau_{j}^{i}(t) \in\left[t_{3}+c_{i} T_{0}^{(i)}+W, t_{3}+T_{0}^{(i)}\right)$. Therefore we have:

$$
\begin{aligned}
c_{i} T_{0}^{(i)}+W & =\left(t_{3}+c_{i} T_{0}^{(i)}+W\right) \bmod T_{0}^{(i)} \\
& \leq\left(t_{3}+c_{i} T_{0}^{(i)}+W\right) \bmod T_{0}^{(i)}+\left(\tau_{j}^{i}(t)-\left(t_{3}+c_{i} T_{0}^{(i)}+W\right)\right) \bmod T_{0}^{(i)} \\
& <\left(t_{3}+c_{i} T_{0}^{(i)}+W\right) \bmod T_{0}^{(i)}+\left(t_{3}+T_{0}^{(i)}-\left(t_{3}+c_{i} T_{0}^{(i)}+W\right)\right) \bmod T_{0}^{(i)} \\
& =T_{0}^{(i)} .
\end{aligned}
$$

Therefore

$c_{i} T_{0}^{(i)}+W \leq\left(t_{3}+c_{i} T_{0}^{(i)}+W\right) \bmod T_{0}^{(i)}+\left(\tau_{j}^{i}(t)-\left(t_{3}+c_{i} T_{0}^{(i)}+W\right)\right) \bmod T_{0}^{(i)}<T_{0}^{(i)}$.

It follows that:

$$
\begin{aligned}
& c_{i} T_{0}^{(i)}+W \\
\leq & \left(t_{3}+c_{i} T_{0}^{(i)}+W\right) \bmod T_{0}^{(i)}+\left(\tau_{j}^{i}(t)-\left(t_{3}+c_{i} T_{0}^{(i)}+W\right)\right) \bmod T_{0}^{(i)} \\
= & {\left[\left(t_{3}+c_{i} T_{0}^{(i)}+W\right) \bmod T_{0}^{(i)}+\left(\tau_{j}^{i}(t)-\left(t_{3}+c_{i} T_{0}^{(i)}+W\right)\right) \bmod T_{0}^{(i)}\right] \bmod T_{0}^{(i)} } \\
= & \tau_{j}^{i}(t) \bmod T_{0}^{(i)} \\
< & T_{0}^{(i)} .
\end{aligned}
$$

From the inequality $c_{i} T_{0}^{(i)}+W \leq \tau_{j}^{i}(t) \bmod T_{0}^{(i)}<T_{0}^{(i)}$ proven above and the definition of $M_{1}^{(i)}(t)$, it follows that $M_{1}^{(i)}\left(\tau_{j}^{i}(t)\right)=0$ for all $t \in\left[t_{2}, t_{2}+W\right)$. We have proven that node $i$ is silent during the interval $\left[t_{w}, t_{w}+W\right.$ ) (wrt to node $j$ 's clock). Therefore there exists a collision-free pulse from node $j$ to node $i$ of length $W$ at $\left[t_{w}, t_{w}+W\right)$ all with respect to node $j$ 's clock.

Now consider case two in which the primary pulse generated by node $i$ collides with a secondary pulse generated by node $j$. We show that there exists a pulse of length $W$ generated by node $j$ that does not collide with node $i$.

Lemma 3.3. Assume $i>j$ and $a_{i j} \leq 1$. Let $I_{2}:=\left[t_{2}, t_{2}+W\right)$ be a primary pulse of $M_{1}^{(j)}(t)$ with respect to node $j$ 's clock and let $I_{3}:=\left[t_{3}, t_{3}+W\right)$ be a secondary pulse of $M_{1}^{(i)}(t)$ with respect to node i's clock. Suppose the two pulses overlap. That is, $\left[t_{2}, t_{2}+W\right) \cap \tau_{i}^{j}\left(\left[t_{3}, t_{3}+W\right)\right) \neq \emptyset$. There exists a pulse at $t_{w}:=t_{2}+c_{j} T_{0}^{(j)}$ with respect to node $j$ 's clock that does not overlap with any pulse of $M_{1}^{(i)}\left(\tau_{j}^{i}(t)\right)$.

Proof. We will show:

(i) $\left[t_{w}, t_{w}+W\right) \subset \tau_{i}^{j}\left(\left[t_{3}+W, t_{3}+\left(1-c_{i}\right) T_{0}^{(i)}\right)\right)$,

(ii) $M_{1}^{(j)}(t)=1$ and $M_{1}^{(i)}\left(\tau_{j}^{i}(t)\right)=0$ for all $t \in\left[t_{w}, t_{w}+W\right)$.

If the primary pulse of node $j$ collides with the secondary pulse of node $i$ at time $t_{2}$ (wrt to node $j$ 's clock) we show that there exists a secondary pulse that does not 
collide with node $i$ at $t_{w}:=t_{2}+c_{j} T_{0}^{(j)}$ with respect to node $j$ 's clock. To prove this, we only need to show that $\left[t_{w}, t_{w}+W\right) \subset \tau_{i}^{j}\left(\left[t_{3}+W, t_{3}+\left(1-c_{i}\right) T_{0}^{(i)}\right)\right)$.

(i) We show that the parameters of the orthogonal MAC code guarantee this property. Given the definition of $c_{j}$ we first show that $t_{w} \geq \tau_{i}^{j}\left(t_{3}+\left(1-c_{i}\right) T_{0}^{(i)}\right)$ due to the following sequence of inequalities:

$$
\begin{array}{ll} 
& 16 n \geq 5 n-j \quad \forall j \in\{1, \ldots, n\}, \\
\Rightarrow \quad & \frac{1}{(5 n-j) a_{\max }} \geq \frac{1}{16 n a_{\max }}, \\
\Rightarrow \quad & c_{j} \geq \frac{1}{16 n a_{\max }}, \\
\Rightarrow \quad & c_{j} \geq \frac{2 W a_{\max }}{32 W n a_{\max }^{2}}, \\
\Rightarrow \quad & c_{j} \geq \frac{2 W a_{\max }}{j T_{0}} \\
\Rightarrow \quad & c_{j} \geq \frac{W}{T_{0}^{(j)}}+\frac{W}{a_{i j} T_{0}^{(j)}}, \\
\Rightarrow \quad & c_{j} T_{0}^{(j)} \geq W+\frac{W}{a_{i j}}, \\
\Rightarrow \quad & c_{j} T_{0}^{(j)} \geq t_{2}+W-t_{2}+\frac{W}{a_{i j}}, \\
\Rightarrow \quad & c_{j} T_{0}^{(j)} \geq \tau_{i}^{j}\left(t_{3}\right)-t_{2}+\frac{W}{a_{i j}}, \\
\Rightarrow \quad & t_{2}+c_{j} T_{0}^{(j)} \geq \tau_{i}^{j}\left(t_{3}\right)+\frac{W}{a_{i j}}, \\
\Rightarrow \quad & t_{w} \geq \tau_{i}^{j}\left(t_{3}+W\right) .
\end{array}
$$

It follows that $t_{w} \geq \tau_{i}^{j}\left(t_{3}+W\right)$. Next we show that our choice of $c_{j}:=\frac{1}{a_{\max }(5 n-j)}$ guarantees that $t_{w}+W<\tau_{i}^{j}\left(t_{3}+\left(1-c_{i}\right) T_{0}^{(i)}\right)$ due to the following sequence of inequalities:

$$
\begin{array}{ll} 
& c_{j}=\frac{1}{(5 n-j) a_{\max }}, \\
\Rightarrow & c_{j}<\frac{11 n^{2} a_{\max }}{16 n^{2} a_{\max }}, \\
\Rightarrow & c_{j}<1+\frac{1}{n}-\frac{1}{4 n a_{m a x}}-\frac{1}{4 n^{2} a_{\max }}-\frac{1}{16 n a_{\max }}, \\
\Rightarrow & c_{j}<1-c_{n}+\frac{1-c_{n}}{n}-\frac{1}{16 n a_{\max }}, \\
\Rightarrow & c_{j}<\left(1-c_{n}\right)+\frac{1-c_{n}}{j}-\frac{2 W}{j a_{i j}\left(32 W n a_{\max }^{2}\right)}, \\
\Rightarrow & c_{j}<\frac{1-c_{n}}{a_{i j}}+\frac{1-c_{n}}{j a_{i j}}-\frac{2 W}{j a_{i j} T_{0}}, \\
\Rightarrow \quad c_{j}<\frac{1-c_{n}}{a_{i j}}+\frac{[(n-j+1)-(n-i+1)]\left(1-c_{n}\right)}{j a_{i j}}-\frac{2 W}{j a_{i j} T_{0}}, \\
\Rightarrow \quad c_{j}<\frac{\left(1-c_{n}\right)}{a_{i j}}\left(\frac{i}{j}\right)-\frac{2 W}{j a_{i j} T_{0}}, \\
\Rightarrow \quad c_{j}<\frac{\left(1-c_{n}\right) T_{0}^{(i)}}{a_{i j} T_{0}^{(j)}}-\frac{2 W}{a_{i j} T_{0}^{(j)}}, \\
\Rightarrow \quad c_{j}<\frac{\left(1-c_{i}\right) T_{0}^{(i)}}{a_{i j} T_{0}^{(j)}}-\frac{W}{a_{i j} T_{0}^{(j)}}-\frac{W}{T_{0}^{(j)}}, \\
\Rightarrow \quad c_{j} T_{0}^{(j)}<\frac{\left(1-c_{i}\right) T_{0}^{(i)}}{a_{i j}}-\frac{W}{a_{i j}}-W, \\
\Rightarrow \quad c_{j} T_{0}^{(j)}<\tau_{i}^{j}\left(t_{3}\right)-\tau_{i}^{j}\left(t_{3}+W\right)+\frac{\left(1-c_{i}\right) T_{0}^{(i)}}{a_{i j}}-W, \\
\Rightarrow \quad c_{j} T_{0}^{(j)}<\tau_{i}^{j}\left(t_{3}\right)-t_{2}+\frac{\left(1-c_{i}\right) T_{0}^{(i)}}{a_{i j}}-W, \\
\Rightarrow \quad t_{2}+c_{j} T_{0}^{(j)}+W<\tau_{i}^{j}\left(t_{3}\right)+\frac{\left(1-c_{i}\right) T_{0}^{(i)}}{a_{i j}}, \\
\Rightarrow \quad t_{w}+W<\tau_{j}^{i}\left(t_{3}+\left(1-c_{i}\right) T_{0}^{(i)}\right) .
\end{array}
$$


It follows that $\left[t_{w}, t_{w}+W\right) \subset\left[\tau_{i}^{j}\left(t_{3}+W\right), \tau_{i}^{j}\left(t_{3}+\left(1-c_{i}\right) T_{0}^{(i)}\right)\right)$.

(ii) Now use part (i) to show that node $j$ transmits during the interval $\left[t_{w}, t_{w}+W\right)$ with respect to node $j$ 's clock while node $i$ is silent. Let $t \in\left[t_{w}, t_{w}+W\right)$. From the definition of $T_{0}^{(j)}$ we have $\left|\left[t_{w}, t_{w}+W\right)\right|<T_{0}^{(j)}$. Now let $t \in\left[t_{w}, t_{w}+W\right)$. From the definition of $t_{2}$ and $t_{w}$ we have:

$$
\begin{aligned}
c_{j} T_{0}^{(j)} & =t_{w} \bmod T_{0}^{(j)} \\
& \leq t_{w} \bmod T_{0}^{(j)}+\left(t-t_{w}\right) \bmod T_{0}^{(j)} \\
& <t_{w} \bmod T_{0}^{(j)}+\left(t_{w}+W-t_{w}\right) \bmod T_{0}^{(j)} \\
& =c_{j} T_{0}^{(j)}+W .
\end{aligned}
$$

Therefore $c_{j} T_{0}^{(j)} \leq t_{w} \bmod T_{0}^{(j)}+\left(t-t_{w}\right) \bmod T_{0}^{(j)}<c_{j} T_{0}^{(j)}+W$. It follows from this inequality that:

$$
\begin{aligned}
c_{j} T_{0}^{(j)} & \leq t_{w} \bmod T_{0}^{(j)}+\left(t-t_{w}\right) \bmod T_{0}^{(j)} \\
& =\left[t_{w} \bmod T_{0}^{(j)}+\left(t-t_{w}\right) \bmod T_{0}^{(j)}\right] \bmod T_{0}^{(j)} \\
& =t \bmod T_{0}^{(j)} \\
& <c_{j} T_{0}^{(j)}+W .
\end{aligned}
$$

From the inequality $c_{j} T_{0}^{(j)} \leq t \bmod T_{0}^{(j)}<c_{j} T_{0}^{(j)}+W$ proven above, and the definition of $M_{1}^{(j)}(t)$, it follows that $M_{1}^{(j)}(t)=1$ for all $t \in\left[t_{w}, t_{w}+W\right)$. So we have proven that node $j$ transmits during the interval $\left[t_{w}, t_{w}+W\right)$. Now we need to show that node $i$ is silent. We have $t \in \tau_{i}^{j}\left(\left[t_{3}+W, t_{3}+\left(1-c_{i}\right) T_{0}^{(i)}\right)\right)$ since $\left[t_{w}, t_{w}+W\right) \subset$ $\tau_{i}^{j}\left(\left[t_{3}+W, t_{3}+\left(1-c_{i}\right) T_{0}^{(i)}\right)\right)$. Therefore $\tau_{j}^{i}(t) \in\left[t_{3}+W, t_{3}+\left(1-c_{i}\right) T_{0}^{(i)}\right)$. Clearly, $\left|\left[t_{3}+W, t_{3}+\left(1-c_{i}\right) T_{0}^{(i)}\right)\right|<T_{0}^{(i)}$. We have:

$$
\begin{aligned}
W+c_{i} T_{0}^{(i)} & =\left(t_{3}+W\right) \bmod T_{0}^{(i)} \\
& \leq\left(t_{3}+W\right) \bmod T_{0}^{(i)}+\left(\tau_{j}^{i}(t)-\left(t_{3}+W\right)\right) \bmod T_{0}^{(i)} \\
& <\left(t_{3}+W\right) \bmod T_{0}^{(i)}+\left(t_{3}+\left(1-c_{i}\right) T_{0}^{(i)}-\left(t_{3}+W\right)\right) \bmod T_{0}^{(i)} \\
& =W+c_{i} T_{0}^{(i)}+\left(1-c_{i} T_{0}^{(i)}-W\right. \\
& =T_{0}^{(i)} .
\end{aligned}
$$

Therefore $W+c_{i} T_{0}^{(i)} \leq\left(t_{3}+W\right) \bmod T_{0}^{(i)}+\left(\tau_{j}^{i}(t)-\left(t_{3}+W\right)\right) \bmod T_{0}^{(i)}<T_{0}^{(i)}$. It follows that:

$$
\begin{aligned}
W+c_{i} T_{0}^{(i)} & \leq\left(t_{3}+W\right) \bmod T_{0}^{(i)}+\left(\tau_{j}^{i}(t)-\left(t_{3}+W\right)\right) \bmod T_{0}^{(i)} \\
& =\leq\left[\left(t_{3}+W\right) \bmod T_{0}^{(i)}+\left(\tau_{j}^{i}(t)-\left(t_{3}+W\right)\right) \bmod T_{0}^{(i)}\right] \bmod T_{0}^{(i)} \\
& =\tau_{j}^{i}(t) \bmod T_{0}^{(i)} \\
& <T_{0}^{(i)} .
\end{aligned}
$$


From the inequality $c_{i} T_{0}^{(i)}+W \leq \tau_{j}^{i}(t) \bmod T_{0}^{(i)}<T_{0}^{(i)}$ proven above and the definition of $M_{1}^{(i)}(t)$, it follows that $M_{1}^{(i)}\left(\tau_{j}^{i}(t)\right)=0$ for all $t \in\left[t_{2}, t_{2}+W\right)$. We have proven that node $i$ is silent during the interval $\left[t_{w}, t_{w}+W\right)$ (wrt to node $j$ 's clock). Therefore there exists a collision-free pulse from node $j$ to node $i$ of length $W$ at the interval $\left[t_{w}, t_{w}+W\right)$ measured by node $j$ 's clock.

Now we prove that node $j$ can successfully transmit a message of length $W$ to node $i$. We show, using the previous three lemmas, that within the interval $\left[t_{j}, t_{j}+T_{j-1,2}\right)$ there exists a pulse of length $W$ generated by node $j$ that does not collide with a pulse generated by node $i$.

Lemma 3.4. Suppose $a_{i j} \leq 1$ and $i>j$. Given $t_{1}$ such that $\tau_{j}^{i}(t) \geq 0$, there exists an interval $\left[t_{w}, t_{w}+W\right)$ that satisfies the following conditions for all $t \in\left[t_{w}, t_{w}+W\right)$ :

(i) $\left[t_{w}, t_{w}+W\right) \subset\left[t_{1}, t_{1}+T_{n, 2}\right)$,

(ii) $\left[\tau_{j}^{i}\left(t_{w}\right), \tau_{j}^{i}\left(t_{w}+W\right)\right) \subset\left[\tau_{j}^{i}\left(t_{1}\right), \tau_{j}^{i}\left(t_{1}\right)+T_{n, 2}\right)$,

(iii) $M_{1}^{(j)}(t) s_{1}^{(j, i)}(t)=1$,

(iv) $\left.M_{1}^{(i)}\left(\tau_{j}^{i}(t)\right) s_{1}^{(i, j)}\left(\tau_{j}^{i}(t)\right)\right)+s_{1}^{(i, j)}\left(\tau_{j}^{i}(t)\right)=1$.

Proof. From Lemma 3.1 there exists an interval $\left[t_{j}, t_{j}+T_{j-1,2}\right)$ that satisfies (10)-(13). Now $\left|\left[t_{j}, t_{j}+T_{j-1,2}\right)\right|<T_{j-1,2}$. Moreover,

$$
\begin{aligned}
T_{j-1,2} & \geq T_{0,2} \\
& =2 T_{n, 1} \\
& =2\left(\left\lceil n a_{\max }\right\rceil+2\right) T_{0}^{(n)} \\
& \geq 2\left(\left\lceil n a_{\max }\right\rceil+2\right) T_{0}^{(j)} .
\end{aligned}
$$

It follows from the last inequality that there exists an interval $I_{2}:=\left[t_{2}, t_{2}+W\right) \subset$ $\left[t_{j}, t_{j}+T_{j-1,2}\right)$ that satisfies the following conditions:

(i) $0 \leq t \bmod T_{0}^{(j)}<W$,

(ii) $0 \leq t_{2}-t_{j}<T_{0}^{(j)}$.

There are three cases that may occur: Case 1: $M_{1}^{(i)}\left(\tau_{j}^{i}(t)\right)=0$, for all $\left[t_{2}, t_{2}+W\right)$. In this case, set $t_{w}:=t_{2}$. It follows from condition (i) and the definition of $M_{1}^{(j)}(t)$, that for all $t \in\left[t_{2}, t_{2}+W\right)$ we have,

$$
\begin{aligned}
M_{1}^{(j)}(t) & =1, \\
M_{1}^{(i)}\left(\tau_{j}^{i}(t)\right) & =0 .
\end{aligned}
$$

In addition, we clearly have $\left[t_{w}, t_{w}+W\right) \subset\left[t_{j}, t_{j}+T_{j-1,2}\right)$. Case 2: $M_{1}^{(i)}\left(\tau_{j}^{i}(t)\right) \neq 0$ for all $t \in\left[t_{2}, t_{2}+W\right)$. More specifically, there exists an interval $I_{3}:=\left[t_{3}, t_{3}+W\right)$ such that $0 \leq t \bmod T_{0}^{(i)}<W$ for all $t \in I_{3}$ and $\left[t_{2}, t_{2}+W\right) \cap\left[\tau_{i}^{j}\left(t_{3}\right), \tau_{i}^{j}\left(t_{3}+W\right)\right) \neq \emptyset$.

In this case, let $k=\left\lfloor\frac{1}{a_{i j}}\left(\frac{i}{j}\right)-\frac{W}{T_{0}^{(j)}}-\frac{W}{a_{i j} T_{0}^{(j)}}-c_{j}\right\rfloor$, and let $t_{w}:=t_{2}+k T_{0}^{(j)}$. It 
follows from Lemma 3.2 that for all $t \in\left[t_{w}, t_{w}+W\right)$ :

$$
\begin{aligned}
M_{1}^{(j)}(t) & =1, \\
M_{1}^{(i)}\left(\tau_{j}^{i}(t)\right) & =0 .
\end{aligned}
$$

Clearly $t_{w} \geq t_{j}$ since $t_{w} \geq t_{2} \geq t_{j}$. We also can show that $t_{w}+W<t_{j}+T_{j-1,2}$ due to the following sequence of inequalities:

$$
\begin{aligned}
t_{w}+W & \leq t_{2}+k T_{0}^{(j)}+W \\
& \leq t_{j}+T_{0}^{(j)}+k T_{0}^{(j)}+W \\
& <t_{j}+T_{0}^{(j)}+k T_{0}^{(j)}+T_{0}^{(j)} \\
& =t_{j}+(k+2) T_{0}^{(j)} \\
& <t_{j}+(k+2) T_{0}^{(n)} \\
& \leq t_{j}+\left(\left\lceil n a_{\max }\right\rceil+2\right) T_{0}^{(n)} \\
& =t_{j}+T_{n, 1} \\
& <t_{j}+T_{j-1,2} .
\end{aligned}
$$

Therefore $\left[t_{w}, t_{w}+W\right) \subset\left[t_{j}, t_{j}+T_{j-1,2}\right)$. Case 3: $M_{1}^{(i)}\left(\tau_{j}^{i}(t)\right) \neq 0$ for all $t \in$ $\left[t_{2}, t_{2}+W\right)$. More specifically, there exists an interval $I_{3}:=\left[t_{3}, t_{3}+W\right)$ such that $c_{i} T_{0}^{(i)} \leq t \bmod T_{0}^{(i)}<c_{i} T_{0}^{(j)}+W$ for all $t \in I_{3}$ and $\left[t_{2}, t_{2}+W\right) \cap\left[\tau_{i}^{j}\left(t_{3}\right), \tau_{i}^{j}\left(t_{3}+W\right)\right) \neq \emptyset$. In this case let $t_{w}:=t_{2}+c_{j} T_{0}^{(j)}$. It follows from Lemma 3.3 that for all $t \in\left[t_{w}, t_{w}+W\right)$ :

$$
\begin{aligned}
M_{1}^{(j)}(t) & =1, \\
M_{1}^{(i)}\left(\tau_{j}^{i}(t)\right) & =0 .
\end{aligned}
$$

Clearly $t_{w} \geq t_{j}$ since $t_{w} \geq t_{2} \geq t_{j}$. We also can show that $t_{w}+W<t_{j}+T_{j-1,2}$ due to the following sequence of inequalities:

$$
\begin{aligned}
t_{w}+W & =t_{2}+c_{j} T_{0}^{(j)}+W \\
& \leq t_{j}+T_{0}^{(j)}+W \\
& <t_{j}+2 T_{0}^{(j)} \\
& <t_{j}+2 T_{0}^{(n)} \\
& <t_{j}+\left(\left\lceil n a_{\max }\right\rceil+2\right) T_{0}^{(n)} \\
& =t_{j}+T_{n, 1} \\
& <t_{j}+T_{0,2} \\
& \leq t_{j}+T_{j-1,2} .
\end{aligned}
$$

Therefore $\left[t_{w}, t_{w}+W\right) \subset\left[t_{j}, t_{j}+T_{j-1,2}\right)$. It follows that $\left[t_{w}, t_{w}+W\right) \subset\left[t_{j}, t_{j}+T_{j-1,2}\right)$ 
in all cases and for all $t \in\left[t_{w}, t_{w}+W\right)$ we have:

$$
\begin{aligned}
M_{1}^{(j)}(t) & =1, \\
M_{1}^{(i)}\left(\tau_{j}^{i}(t)\right) & =0, \\
s_{1}^{(j, i)}(t) & =1, \\
s_{1}^{(i, j)}\left(\tau_{j}^{i}(t)\right) & =1 .
\end{aligned}
$$

where the latter two equalities follow from Lemma 3.1 cited at the onset of the proof. Therefore for all $t \in\left[t_{w}, t_{w}+W\right)$ we have:

$$
\begin{aligned}
M_{1}^{(j)}(t) s_{1}^{(j, i)}(t) & =1, \\
M_{1}^{(i)}\left(\tau_{j}^{i}(t)\right) s_{1}^{(i, j)}\left(\tau_{j}^{1}(t)\right)+s_{1}^{(i, j)}\left(\tau_{j}^{i}(t)\right) & =1 .
\end{aligned}
$$

Now we repeat the process when $a_{i j}>1$. In the following lemma we show that for any pair of nodes $i$ and $j$, there exists an interval of time, $\left[t_{j}, t_{j}+T_{j-1,2}\right)$ with respect to node $j$ 's clock, in which node $i$ and $j$ are scheduled to communicate with each other using the signals $M_{2}^{(i)}(t)$ and $M_{2}^{(j)}(t)$ respectively.

Lemma 3.5. Without loss of generality assume $i>j$. There exists an interval $\left[t_{j}, t_{j}+T_{j-1,2}\right)$ with respect to node $j$ 's clock in which node $i$ and $j$ are scheduled to communicate with each other using the signals $M_{2}^{(i)}(t)$ and $M_{2}^{(j)}(t)$ respectively. That is, given $t_{1}$ such that $\tau_{j}^{i}\left(t_{1}\right) \geq 0$, the interval $\left[t_{j}, t_{j}+T_{j-1,2}\right)$ satisfies the following conditions:

$$
\begin{aligned}
{\left[t_{j}, t_{j}+T_{j-1,2}\right) } & \subset\left[t_{1}, t_{1}+T_{n, 2}\right), \\
\tau_{j}^{i}\left(\left[t_{j}, t_{j}+T_{j-1,2}\right)\right) & \subset\left[\tau_{j}^{i}\left(t_{1}\right), \tau_{j}^{i}\left(t_{1}\right)+T_{n, 2}\right), \\
s_{2}^{(i, j)}\left(\tau_{j}^{i}(t)\right) & =1, \forall t \in\left[t_{j}, t_{j}+T_{j-1,2}\right), \\
s_{2}^{(j, i)}(t) & =1, \forall t \in\left[t_{j}, t_{j}+T_{j-1,2}\right) .
\end{aligned}
$$

Proof. The proof of this lemma is very similar to the proof of Lemma 3.1.

First, we consider case one in which a primary pulse generated by node $j$ collides with a primary pulse generated by node $i$. We show that there exists a pulse of length $W$ generated by node $j$ that does not collide with node $i$.

LemMa 3.6. Assume $i>j$ and $a_{i j}>1$. Let $I_{3}:=\left[t_{3}, t_{3}+W\right)$ be a primary pulse of $M_{2}^{(i)}(t)$ with respect to node $i$ 's clock and let $I_{2}:=\left[t_{2}, t_{2}+W\right)$ be a primary pulse of $M_{2}^{(j)}(t)$ with respect to node $j$ 's clock. Suppose the two pulses overlap. That is, $\left[t_{2}, t_{2}+W\right) \cap \tau_{i}^{j}\left(\left[t_{3}, t_{3}+W\right)\right) \neq \emptyset$. There exists a pulse at $t_{w}:=t_{2}+c_{n-j+1} T_{0}^{(n-j+1)}$ with respect to node $j$ 's clock that does not overlap with any pulse of $M_{2}^{(i)}\left(\tau_{j}^{i}(t)\right)$.

Proof. Let $\tilde{i}:=n-i+1$ and $\tilde{j}:=n-j+1$. Then $I_{3}$ is a primary pulse of $M_{1}^{(\tilde{i})}(t)$ and $I_{2}$ is a primary pulse of $M_{1}^{(\tilde{j})}(t)$. Set $a_{\tilde{i} \tilde{j}}:=a_{i j}$. Therefore $a_{\tilde{j} \tilde{i}} \leq 1$. We need to 
show that there exists a pulse at $t_{w}:=t_{2}+c_{\tilde{j}} T_{0}^{(\tilde{j})}$ with respect to node $\tilde{j}$ 's clock that does not overlap with any pulse of $M_{1}^{(\tilde{i})}\left(\tau_{\tilde{j}}^{\tilde{i}}(t)\right)$. This result will be shown in Lemma 3.10 .

Now we consider case two in which a primary pulse generated by node $j$ collides with a secondary pulse generated by node $i$. We show that there exists a pulse of length $W$ generated by node $j$ that does not collide with node $i$.

Lemma 3.7. Assume $i>j$ and $a_{i j}>1$. Let $I_{3}:=\left[t_{3}, t_{3}+W\right)$ be a secondary pulse of $M_{2}^{(i)}(t)$ with respect to node $i$ 's clock, and let $I_{2}:=\left[t_{2}, t_{2}+W\right)$ be a primary pulse of $M_{2}^{(j)}(t)$ with respect to node $j$ 's clock. Suppose the two pulses overlap. That is, $\left[t_{2}, t_{2}+W\right) \cap \tau_{i}^{j}\left(\left[t_{3}, t_{3}+W\right)\right) \neq \emptyset$. There exists a pulse at $t_{w}:=t_{2}+c_{n-j+1} T_{0}^{(n-j+1)}$ with respect to node $j$ 's clock that does not overlap with any pulse of $M_{2}^{(i)}\left(\tau_{j}^{i}(t)\right)$.

Proof. Let $\tilde{i}:=n-i+1$ and $\tilde{j}:=n-j+1$. Then $I_{3}$ is a secondary pulse of $M_{1}^{(\tilde{i})}(t)$ and $I_{2}$ is a primary pulse of $M_{1}^{(\tilde{j})}(t)$. Set $a_{\tilde{i} \tilde{j}}:=a_{i j}$. Therefore $a_{\tilde{j} \tilde{i}} \leq 1$. We need to show that there exists a pulse at $t_{w}:=t_{2}+c_{\tilde{j}} T_{0}^{(\tilde{j})}$ with respect to node $\tilde{j}$ 's clock that does not overlap with any pulse of $M_{1}^{(\tilde{i})}\left(\tau_{\tilde{j}}^{\tilde{i}}(t)\right)$. This result will be shown in Lemma 3.11.

Now we prove that node $j$ can successfully transmit a message of length $W$ to node $i$ when $a_{i j}>1$. We show, using the previous three lemmas, Lemma 3.5, Lemma 3.7, and Lemma 3.6, that within the interval $\left[t_{j}, t_{j}+T_{j-1,2}\right)$ there exists a pulse of length $W$ generated by node $j$ that does not collide with a pulse generated by node $i$.

Lemma 3.8. Suppose $a_{i j}>1$ and $i>j$. Given $t_{1}$ such that $\tau_{j}^{i}\left(t_{1}\right) \geq 0$, there exists an interval $\left[t_{w}, t_{w}+W\right)$ that satisfies the following conditions for all $t \in\left[t_{w}, t_{w}+W\right)$ :

(i) $\left[t_{w}, t_{w}+W\right) \subset\left[t_{1}, t_{1}+T_{n, 2}\right)$,

(ii) $\left[\tau_{j}^{i}\left(t_{w}\right), \tau_{j}^{i}\left(t_{w}+T_{n, 2}\right)\right) \subset\left[\tau_{j}^{i}\left(t_{1}\right), \tau_{j}^{i}\left(t_{1}\right)+T_{n, 2}\right)$,

(iii) $M_{2}^{(j)}(t) s_{2}^{(j, i)}(t)=1$,

(iv) $M_{2}^{(i)}\left(\tau_{j}^{i}(t)\right) s_{2}^{(i, j)}\left(\tau_{j}^{i}(t)\right)+s_{2}^{(i, j)}\left(\tau_{j}^{i}(t)\right)=1$.

We have proved that node $j$ can transmit a message of length $W$ to node $i$ if $a_{i j} \leq 1$ and if $a_{i j}>1$. Putting both cases together gives us our first theorem; the orthogonal MAC code enables node $j$ to successfully transmit a message of length $W$ units to node $i$ within a finite time $T_{M A C}(W):=T_{n, 2}$ as long as the relative skew $a_{i j} \leq a_{\max }$.

TheOREm 3.1. Without loss of generality, assume $i>j$. Assume there exists $t_{1}$ such that $\tau_{j}^{i}\left(t_{1}\right)>0$. There exists an interval $\left[t_{w}, t_{w}+W\right)$ that satisfies the following conditions for all $t \in\left[t_{w}, t_{w}+W\right)$ and some $k \in\{1,2\}$ :

(i) $\left[t_{w}, t_{w}+W\right) \subset\left[t_{1}, t_{1}+T_{n, 2}\right)$,

(ii) $\left[\tau_{j}^{i}\left(t_{w}\right), \tau_{j}^{i}\left(t_{w}+W\right)\right) \subset\left[\tau_{j}^{i}\left(t_{1}\right), \tau_{j}^{i}\left(t_{1}\right)+T_{n, 2}\right)$,

(iii) $M_{k}^{(i)}\left(\tau_{j}^{i}(t)\right) s_{k}^{(i, j)}\left(\tau_{j}^{i}(t)\right)+s_{k}^{(i, j)}\left(\tau_{j}^{i}(t)\right)=1$,

(iv) $M_{k}^{(j)}(t) s_{k}^{(j, i)}(t)=1$. 
Proof. There are two cases to consider:

Case 1: $a_{i j} \leq 1$. It follows from Lemma 3.4 that there exists an interval $\left[t_{w}, t_{w}+\right.$ $W$ ) that satisfies the following conditions for all $t \in\left[t_{w}, t_{w}+W\right)$ :

(i) $\left[t_{w}, t_{w}+W\right) \subset\left[t_{1}, t_{1}+T_{n, 2}\right)$,

(ii) $\left[\tau_{j}^{i}\left(t_{w}\right), \tau_{j}^{i}\left(t_{w}+W\right)\right) \subset\left[\tau_{j}^{i}\left(t_{1}\right), \tau_{j}^{i}\left(t_{1}\right)+T_{n, 2}\right)$,

(iii) $M_{1}^{(i)}\left(\tau_{j}^{i}(t)\right) s_{1}^{(i, j)}\left(\tau_{j}^{i}(t)\right)+s_{1}^{(i, j)}\left(\tau_{j}^{i}(t)\right)=1$,

(iv) $M_{1}^{(j)}(t) s_{1}^{(j, i)}(t)=1$.

Case 2: $a_{i j}>1$. It follows from Lemma 3.8 that there exists an interval $\left[t_{w}, t_{w}+\right.$ $W)$ that satisfies the following conditions for all $t \in\left[t_{w}, t_{w}+W\right)$ :

(i) $\left[t_{w}, t_{w}+W\right) \subset\left[t_{1}, t_{1}+T_{n, 2}\right)$,

(ii) $\left[\tau_{j}^{i}\left(t_{w}\right), \tau_{j}^{i}\left(t_{w}+W\right)\right) \subset\left[\tau_{j}^{i}\left(t_{1}\right), \tau_{j}^{i}\left(t_{1}\right)+T_{n, 2}\right)$,

(iii) $M_{2}^{(i)}\left(\tau_{j}^{i}(t)\right) s_{2}^{(i, j)}\left(\tau_{j}^{i}(t)\right)+s_{2}^{(i, j)}\left(\tau_{j}^{i}(t)\right)=1$,

(iv) $M_{2}^{(j)}(t) s_{2}^{(j, i)}(t)=1$.

We now show that node $i$ can transmit a message of size $W$ to node $j$. Suppose $i>j$ and $a_{i j} \leq 1$. Consider two nodes $i$ and $j$, where $i>j$ and $a_{i j} \leq 1$. In the following lemma we show that for any two pairs of nodes $i$ and $j$, there exists an interval of time, $\left[t_{i}, t_{i}+T_{i}\right)$, with respect to node $i$ 's clock, in which node $i$ and $j$ are scheduled to communicate with each other using the signals $M_{1}^{(i)}(t)$ and $M_{1}^{(j)}(t)$ respectively.

Lemma 3.9. Suppose $i>j$. Given $t_{1}$ such that $\tau_{i}^{j}\left(t_{1}\right) \geq 0$, there exists an interval $\left[t_{i}, t_{i}+T_{i}\right)$ that satisfies the following conditions for all $t \in\left[t_{i}, t_{i}+T_{i}\right)$ :

$$
\begin{aligned}
{\left[t_{i}, t_{i}+T_{i}\right) } & \subset\left[t_{1}, t_{1}+T_{n, 2}\right), \\
\tau_{i}^{j}\left(\left[t_{i}, t_{i}+T_{i}\right)\right) & \subset\left[\tau_{i}^{j}\left(t_{1}\right), \tau_{i}^{j}\left(t_{1}\right)+T_{n, 2}\right), \\
s_{1}^{(j, i)}\left(\tau_{i}^{j}(t)\right) & =1, \\
s_{1}^{(i, j)}(t) & =1 .
\end{aligned}
$$

Proof. Since $0 \leq t_{1} \bmod 2(n-1) T_{i-1,2}<2(n-1) T_{i-1,2}$, there exists some $t_{a}$, $0 \leq t_{a}<2(n-1) T_{i-1,2}$ such that $\left(t_{1}+t_{a}\right) \bmod 2(n-1) T_{i-1,2}=(j-1) T_{i-1,2}$ if $1 \leq j \leq n-1$. Let $\tilde{t}_{i}:=t_{1}+t_{a}$. It follows from the definition of $s_{1}^{(i, j)}(t)$ in $(6)$ that (39) is satisfied for all $t \in\left[\tilde{t}_{i}, \tilde{t}_{i}+T_{i-1,2}\right)$.

Now since $0 \leq \tau_{i}^{j}\left(\tilde{t}_{i}\right) \bmod 2(n-1) T_{j-1,2}<2(n-1) T_{j-1,2}$, there exists some $t_{b}$, $0 \leq t_{b}<2(n-1) T_{j-1,2}$ such that $\left(\tau_{i}^{j}\left(\tilde{t}_{i}\right)+t_{b}\right) \bmod 2(n-1) T_{j-1,2}=(i-2) T_{j-1,2}$, if $2 \leq i \leq n$. Set $t_{j}:=\tau_{i}^{j}\left(\tilde{t}_{i}\right)+t_{b}$. It follows from the definition of $s_{1}^{(j, i)}(t)$ that (38) is satisfied for all $t \in\left[\tau_{j}^{i}\left(t_{j}\right), \tau_{j}^{i}\left(t_{j}+T_{j-1,2}\right)\right)$. We will prove (36) and show that $\left[t_{i}, t_{i}+T_{i}\right) \subset\left[t_{1}, t_{1}+T_{n, 2}\right)$. Now set $t_{i}:=\tau_{j}^{i}\left(t_{j}\right)$, and let $T_{i}:=\tau_{j}^{i}\left(t_{j}+T_{j-1,2}\right)-\tau_{j}^{i}\left(t_{j}\right)=$ $a_{i j} T_{j-1,2}$. First we show that $t_{i} \geq t_{1}$ :

$$
t_{i}:=\tau_{j}^{i}\left(t_{j}\right)
$$




$$
\begin{aligned}
& =\tau_{j}^{i}\left(\tau_{i}^{j}\left(\tilde{t}_{i}\right)+t_{b}\right) \\
& =\tau_{j}^{i}\left(\tau_{i}^{j}\left(t_{1}+t_{a}\right)+t_{b}\right) \\
& =t_{1}+t_{a}+a_{i j} t_{b} \\
& \geq t_{1} .
\end{aligned}
$$

Next we show that $t_{i}+T_{i}<t_{1}+T_{n, 2}$ :

$$
\begin{aligned}
t_{i}+T_{i} & =t_{1}+t_{a}+a_{i j} t_{b}+T_{i} \\
& <t_{1}+2(n-1) T_{i-1,2}+a_{i j} 2(n-1) T_{j-1,2}+a_{i j} T_{j-1,2} \\
& =t_{1}+2(n-1) T_{i-1,2}+a_{i j} 2 n T_{j-1,2} \\
& =t_{1}+2(n-1) T_{i-1,2}+T_{j, 2} \\
& \leq t_{1}+2(n-1) T_{i-1,2}+T_{i-1,2} \\
& \leq t_{1}+2 n T_{i-1,2} \\
& \leq t_{1}+T_{i, 2} \\
& \leq t_{1}+T_{n, 2} .
\end{aligned}
$$

It follows from both inequalities that $\left[t_{i}, t_{i}+T_{i}\right) \subset\left[t_{1}, t_{1}+T_{n, 2}\right)$. Therefore (36) is proved. Now we prove (37) and show that $\left[\tau_{i}^{j}\left(t_{i}\right), \tau_{i}^{j}\left(t_{i}+T_{i}\right)\right) \subset\left[\tau_{i}^{j}\left(t_{1}\right), \tau_{i}^{j}\left(t_{1}\right)+T_{n, 2}\right)$. First we show that $\tau_{i}^{j}\left(t_{i}\right) \geq \tau_{i}^{j}\left(t_{1}\right)$ due to the following series of inequalities:

$$
\begin{aligned}
\tau_{i}^{j}\left(t_{i}\right) & =\tau_{i}^{j}\left(\tau_{j}^{i}\left(t_{j}\right)\right) \\
& =t_{j} \\
& =\tau_{i}^{j}\left(\tilde{t}_{i}\right)+t_{b} \\
& =\tau_{i}^{j}\left(t_{1}+t_{a}\right)+t_{b} \\
& =\tau_{i}^{j}\left(t_{1}\right)+a_{j i} t_{a}+t_{b} \\
& \geq \tau_{i}^{j}\left(t_{1}\right) .
\end{aligned}
$$

Next we show that $\tau_{i}^{j}\left(t_{i}+T_{i}\right)<\tau_{i}^{j}\left(t_{1}\right)+T_{n, 2}$ due to the following series of inequalities:

$$
\begin{aligned}
\tau_{i}^{j}\left(t_{i}+T_{i}\right) & =\tau_{i}^{j}\left(t_{i}\right)+a_{j i} T_{i} \\
& =\tau_{i}^{j}\left(t_{1}\right)+a_{j i} t_{a}+t_{b}+a_{j i}\left(a_{i j} T_{j-1,2}\right) \\
& <\tau_{i}^{j}\left(t_{1}\right)+a_{\max } 2(n-1) T_{i-1,2}+2(n-1) T_{j-1,2}+T_{j-1,2} \\
& =\tau_{i}^{j}\left(t_{1}\right)+a_{\max } 2(n-1) T_{i-1,2}+2 n T_{j-1,2} \\
& \leq \tau_{i}^{j}\left(t_{1}\right)+a_{\max } 2(n-1) T_{i-1,2}+T_{j, 2}
\end{aligned}
$$




$$
\begin{aligned}
& \leq \tau_{i}^{j}\left(t_{1}\right)+a_{\max } 2(n-1) T_{i-1,2}+T_{i-1,2} \\
& \leq \tau_{i}^{j}\left(t_{1}\right)+a_{\max } 2 n T_{i-1,2} \\
& =\tau_{i}^{j}\left(t_{1}\right)+T_{i, 2} \\
& \leq \tau_{i}^{j}\left(t_{1}\right)+T_{n, 2} .
\end{aligned}
$$

It follows that $\left[\tau_{i}^{j}\left(t_{i}\right), \tau_{i}^{j}\left(t_{i}+T_{i}\right)\right) \subset\left[\tau_{i}^{j}\left(t_{1}\right), \tau_{i}^{j}\left(t_{1}\right)+T_{n, 2}\right)$. Therefore (37) is satisfied.

Now we again consider two separate cases. First, we consider case one in which a primary pulse generated by node $i$ collides with a primary pulse generated by node $j$. We show that there exists a pulse of length $W$ generated by node $i$ that does not collide with node $j$.

Lemma 3.10. Assume $i>j$ and $a_{i j} \leq 1$. Let $I_{2}:=\left[t_{2}, t_{2}+W\right)$ be a primary pulse of $M_{1}^{(j)}(t)$ with respect to node $j$ 's clock and let $I_{3}:=\left[t_{3}, t_{3}+W\right)$ be a primary pulse of $M_{1}^{(i)}(t)$ with respect to node $i$ 's clock. Suppose the two pulses overlap. That is, $\left[t_{3}, t_{3}+W\right) \cap \tau_{j}^{i}\left(\left[t_{2}, t_{2}+W\right)\right) \neq \emptyset$. There exists a pulse at $t_{w}:=t_{3}+c_{i} T_{0}^{(i)}$ with respect to node $i$ 's clock that does not overlap with any pulse of $M_{1}^{(j)}\left(\tau_{i}^{j}(t)\right)$.

Proof. We will show:

(i) $\left[t_{w}, t_{w}+W\right) \subset \tau_{j}^{i}\left(\left[t_{2}+c_{j} T_{0}^{(j)}+W, t_{2}+T_{0}^{(j)}\right)\right)$,

(ii) $M_{1}^{(i)}(t)=1$ and $M_{1}^{(j)}\left(\tau_{i}^{j}(t)\right)=0$ for all $t \in\left[t_{w}, t_{w}+W\right)$.

If the primary pulse of node $i$ collides with the primary pulse of node $j$ at time $t_{3}$ (wrt to node $i$ 's clock) we show that there exists a secondary pulse that does not collide with node $i$ at time $t_{w}:=t_{3}+c_{i} T_{0}^{(i)}$. We will first show that $\left[t_{w}, t_{w}+W\right) \subset$ $\left.\tau_{j}^{i}\left(\left[t_{2}+c_{j} T_{0}^{(j)}+W\right), t_{2}+T_{0}^{(j)}\right)\right)$ and then show in (ii) that this condition proves existence of a collision-free secondary pulse.

(i) We show that the parameters of the orthogonal MAC code selected at the onset guarantee that $\left[t_{w}, t_{w}+W\right) \subset \tau_{j}^{i}\left(\left[t_{2}+c_{j} T_{0}^{(j)}+W, t_{2}+T_{0}^{(j)}\right)\right)$. First we show that our selection of $T_{0}:=32 W n a_{\text {max }}^{2}$ guarantees that $t_{w} \geq \tau_{j}^{i}\left(t_{2}+c_{j} T_{0}^{(j)}+W\right)$ due to the following sequence of inequalities:

$$
\begin{aligned}
& T_{0} \geq 10 n W a_{\max }, \\
\Rightarrow & \frac{T_{0}}{5 n} \geq 2 W a_{\max }, \\
\Rightarrow & \frac{T_{0}}{5 n} \geq \frac{W}{a_{i j}}+W, \\
\Rightarrow & \left(\frac{5 n}{(5 n)(5 n)}\right) T_{0} \geq \frac{W}{a_{i j}}+W, \\
\Rightarrow & \left(\frac{5 n}{(5 n-i)(5 n-j)}\right) T_{0} \geq \frac{W}{a_{i j}}+W, \\
\Rightarrow & \left(\frac{5 n(i-j)}{(5 n-i)(5 n-j)}\right) T_{0} \geq \frac{W}{a_{i j}}+W, \\
\Rightarrow & \left(\frac{5 n i-i j-5 n j+i j}{(5 n-i)(5 n-j)}\right) T_{0} \geq \frac{W}{a_{i j}}+W, \\
\Rightarrow & \left(\frac{i(5 n-j)-j(5 n-i)}{(5 n-i)(5 n-j)}\right) T_{0} \geq \frac{W}{a_{i j}}+W, \\
\Rightarrow & \left(\frac{i}{5 n-i}-\frac{j}{5 n-j}\right) T_{0} \geq \frac{W}{a_{i j}}+W,
\end{aligned}
$$




$$
\begin{aligned}
& \Rightarrow \quad\left(i c_{i}-j c_{j}\right) T_{0} \geq \frac{W}{a_{i j}}+W \\
& \Rightarrow \quad c_{i} i T_{0}-c_{j} j T_{0} \geq \frac{W}{a_{i j}}+W \\
& \Rightarrow \quad c_{i} T_{0}^{(i)}-c_{j} T_{0}^{(j)} \geq \frac{W}{a_{i j}}+W \\
& \Rightarrow \quad \frac{c_{i} T_{0}^{(i)}}{a_{i j}}-c_{j} T_{0}^{(j)} \geq \frac{W}{a_{i j}}+W \\
& \Rightarrow \quad c_{i} T_{0}^{(i)}-a_{i j} c_{j} T_{0}^{(j)} \geq W+a_{i j} W \\
& \Rightarrow \quad c_{i} T_{0}^{(i)}-a_{i j} c_{j} T_{0}^{(j)} \geq \tau_{j}^{i}\left(t_{2}\right)-t_{3}+a_{i j} W \\
& \Rightarrow \quad t_{3}+c_{i} T_{0}^{(i)} \geq \tau_{j}^{i}\left(t_{2}\right)+a_{i j} c_{j} T_{0}^{(j)}+a_{i j} W \\
& \Rightarrow \quad t_{w} \geq \tau_{j}^{i}\left(t_{2}\right)+a_{i j} c_{j} T_{0}^{(j)}+a_{i j} W \\
& \Rightarrow \quad t_{w} \geq \tau_{j}^{i}\left(t_{2}+c_{j} T_{0}^{(j)}+W\right) .
\end{aligned}
$$

Next we show that our choice $c_{j}$ and $T_{0}^{(j)}$ guarantees that $\tau_{i}^{j}\left(t_{w}+W\right)<t_{2}+T_{0}^{(j)}$ due to the following sequence of inequalities:

$$
\begin{array}{ll} 
& 1<i 4 a_{\max } \\
\Rightarrow & 2 W a_{\max }<i 8 W a_{\max }^{2}, \\
\Rightarrow & 2 W a_{\max }<i\left(32 W n a_{\max }^{2}\right)\left(\frac{1}{4 n}\right), \\
\Rightarrow & 2 W a_{\max }<T_{0}^{(i)}\left(\frac{4 n-1}{4 n}\right), \\
\Rightarrow & \frac{2 W}{a_{i j}}<T_{0}^{(i)}\left(1-\frac{a_{\max }}{4 n a_{\max }}\right), \\
\Rightarrow & \frac{2 W}{a_{i j}}<T_{0}^{(i)}\left(1-\frac{1}{4 n a_{\max }}\left(\frac{1}{a_{i j}}\right)\right), \\
\Rightarrow & \frac{2 W}{a_{i j}}<T_{0}^{(i)}\left(1-\frac{c_{n}}{a_{i j}}\right), \\
\Rightarrow & W+\frac{W}{a_{i j}}<T_{0}^{(i)}\left(1-\frac{c_{i}}{a_{i j}}\right), \\
\Rightarrow & W+\frac{W}{a_{i j}}<T_{0}^{(i)}-\frac{c_{i} T_{0}^{(i)}}{a_{i j}}, \\
\Rightarrow & W+\frac{W}{a_{i j}}<T_{0}^{(j)}-\frac{c_{i} T_{0}^{(i)}}{a_{i j}}, \\
\Rightarrow & a_{i j} W+W<a_{i j} T_{0}^{(j)}-c_{i} T_{0}^{(i)}, \\
\Rightarrow & \tau_{j}^{i}\left(t_{2}+W\right)-\tau_{j}^{i}\left(t_{2}\right)+W<a_{i j} T_{0}^{(j)}-c_{i} T_{0}^{(i)}, \\
\Rightarrow & t_{3}-\tau_{j}^{i}\left(t_{2}\right)+W<a_{i j} T_{0}^{(j)}-c_{i} T_{0}^{(i)}, \\
\Rightarrow & t_{3}+c_{i} T_{0}^{(i)}+W<\tau_{j}^{i}\left(t_{2}\right)+a_{i j} T_{0}^{(i)}, \\
\Rightarrow & \left.t_{w}+W<\tau_{j}^{i}\left(t_{2}\right)+a_{i j} T_{0}^{(j)}\right), \\
\Rightarrow & t_{w}+W<\tau_{j}^{i}\left(t_{2}+T_{0}^{(i)}\right) .
\end{array}
$$

It follows from both series of inequalities that

$$
\left[t_{w}, t_{w}+W\right) \subset \tau_{j}^{i}\left(\left[t_{2}+c_{j} T_{0}^{(j)}+W, t_{2}+T_{0}^{(j)}\right)\right) .
$$

(ii) Now use part (i) to show that node $i$ transmits during the interval $\left[t_{w}, t_{w}+W\right)$ with respect to node $i$ 's clock while node $j$ is silent. Let $t \in\left[t_{w}, t_{w}+W\right)$. From the definition of $t_{3}$ and $t_{w}$ we have:

$$
c_{i} T_{0}^{(i)}=t_{w} \bmod T_{0}^{(i)}
$$




$$
\begin{aligned}
& \leq t_{w} \bmod T_{0}^{(i)}+\left(t-t_{w}\right) \bmod T_{0}^{(i)} \\
& <c_{i} T_{0}^{(i)}+\left(t_{3}+c_{i} T_{0}^{(i)}+W-\left(t_{3}+c_{i} T_{0}^{(i)}\right)\right) \bmod T_{0}^{(i)} \\
& =c_{i} T_{0}^{(i)}+W
\end{aligned}
$$

Therefore $c_{i} T_{0}^{(i)} \leq t_{w} \bmod T_{0}^{(i)}+\left(t-t_{w}\right) \bmod T_{0}^{(i)}<c_{i} T_{0}^{(i)}+W$. It follows from this inequality that:

$$
\begin{aligned}
c_{i} T_{0}^{(i)} & \leq t_{w} \bmod T_{0}^{(i)}+\left(t-t_{w}\right) \bmod T_{0}^{(i)} \\
& =\left[t_{w} \bmod T_{0}^{(i)}+\left(t-t_{w}\right) \bmod T_{0}^{(i)}\right] \bmod T_{0}^{(i)} \\
& =t \bmod T_{0}^{(i)} \\
& <c_{i} T_{0}^{(i)}+W .
\end{aligned}
$$

Therefore $c_{i} T_{0}^{(i)} \leq t \bmod T_{0}^{(i)}<c_{i} T_{0}^{(i)}+W$ for all $t \in\left[t_{w}, t_{w}+W\right)$. It follows from this inequality and the definition of $M_{1}^{(i)}(t)$, that $M_{1}^{(i)}(t)=1$ for all $t \in\left[t_{w}, t_{w}+W\right)$. So we have shown that node $i$ transmits during the interval $\left[t_{w}, t_{w}+W\right)$. It now remains to show that node $j$ is silent.

We have $\tau_{i}^{j}(t) \in\left[t_{2}+c_{j} T_{0}^{(j)}+W, t_{2}+T_{0}^{(j)}\right)$ since $\tau_{i}^{j}\left(\left[t_{w}, t_{w}+W\right)\right) \subset\left[t_{2}+c_{j} T_{0}^{(j)}+\right.$ $\left.W, t_{2}+T_{0}^{(j)}\right)$. It follows from the definition of $t_{2}$ that:

$$
\begin{aligned}
c_{j} T_{0}^{(j)}+W & =\left(t_{2}+c_{j} T_{0}^{(j)}+W\right) \bmod T_{0}^{(j)} \\
& \leq\left(t_{2}+c_{j} T_{0}^{(j)}+W\right) \bmod T_{0}^{(j)}+\left(\tau_{i}^{j}(t)-\left(t_{2}+c_{j} T_{0}^{(j)}+W\right)\right) \bmod T_{0}^{(j)} \\
& <c_{j} T_{0}^{(j)}+W+\left(t_{2}+T_{0}^{(j)}-\left(t_{2}+c_{j} T_{0}^{(j)}+W\right)\right) \\
& =T_{0}^{(j)} .
\end{aligned}
$$

Therefore

$c_{j} T_{0}^{(j)}+W \leq\left(t_{2}+c_{j} T_{0}^{(j)}+W\right) \bmod T_{0}^{(j)}+\left(\tau_{i}^{j}(t)-\left(t_{2}+c_{j} T_{0}^{(j)}+W\right)\right) \bmod T_{0}^{(j)}<T_{0}^{(j)}$.

It follows from this inequality that:

$$
\begin{aligned}
& c_{j} T_{0}^{(j)}+W \\
\leq & \left(t_{2}+c_{j} T_{0}^{(j)}+W\right) \bmod T_{0}^{(j)}+\left(\tau_{i}^{j}(t)-\left(t_{2}+c_{j} T_{0}^{(j)}+W\right)\right) \bmod T_{0}^{(j)} \\
= & {\left[\left(t_{2}+c_{j} T_{0}^{(j)}+W\right) \bmod T_{0}^{(j)}+\left(\tau_{i}^{j}(t)-\left(t_{2}+c_{j} T_{0}^{(j)}+W\right)\right) \bmod T_{0}^{(j)}\right] \bmod T_{0}^{(j)} } \\
= & \tau_{i}^{j}(t) \bmod T_{0}^{(j)} \\
< & T_{0}^{(j)} .
\end{aligned}
$$

Therefore $c_{j} T_{0}^{(j)}+W \leq \tau_{i}^{j}(t)<T_{0}^{(j)}$ for all $t \in\left[t_{w}, t_{w}+W\right)$. It follows from this fact, and the definition of $M_{1}^{(j)}(t)$, that $M_{1}^{(j)}\left(\tau_{i}^{j}(t)\right)=0$ for all $t \in\left[t_{w}, t_{w}+W\right)$.

Next we consider case two in which a primary pulse generated by node $i$ collides with a secondary pulse generated by node $j$. We show that there exists a pulse of length $W$ that does not collide with node $j$. 
Lemma 3.11. Assume $i>j$ and $a_{i j} \leq 1$. Let $I_{2}:=\left[t_{2}, t_{2}+W\right)$ be a primary pulse of $M_{1}^{(j)}(t)$ with respect to node $j$ 's clock and let $I_{3}:=\left[t_{3}, t_{3}+W\right)$ be a secondary pulse of $M_{1}^{(i)}(t)$ with respect to node $i$ 's clock. Suppose the two pulses overlap. That is, $\left[t_{3}, t_{3}+W\right) \cap \tau_{j}^{i}\left(\left[t_{2}, t_{2}+W\right)\right) \neq \emptyset$. Then there exists a pulse at $t_{w}:=t_{3}+c_{i} T_{0}^{(i)}$ with respect to node $i$ 's clock that does not overlap with any pulse of $M_{1}^{(j)}\left(\tau_{i}^{j}(t)\right)$.

Proof. We will show that:

(i) $\left[t_{w}, t_{w}+W\right) \subset \tau_{j}^{i}\left(\left[t_{2}+W, t_{2}+\left(1-c_{j}\right) T_{0}^{(j)}\right)\right)$,

(ii) $M_{1}^{(i)}(t)=1$ and $M_{1}^{(j)}\left(\tau_{i}^{j}(t)\right)=0$ for all $t \in\left[t_{w}, t_{w}+W\right)$.

If the primary pulse of node $i$ collides with the secondary pulse of node $j$ at time $t_{3}$ (wrt to node $i$ 's clock) we show that there exists a secondary pulse that does not collide with node $j$ at time $t_{w}:=t_{3}+c_{i} T_{0}^{(i)}$. We will first show that $\left[t_{w}, t_{w}+W\right) \subset$ $\left[\tau_{j}^{i}\left(t_{2}+W\right), \tau_{j}^{i}\left(t_{2}+\left(1-c_{j}\right) T_{0}^{(j)}\right)\right)$, and then show in (ii) that this condition proves the existence of a collision-free secondary pulse. (i) We show that the parameters of the orthogonal MAC code selected at the onset guarantee that $\left[t_{w}, t_{w}+W\right) \subset$ $\left[\tau_{j}^{i}\left(t_{2}+W\right), \tau_{j}^{i}\left(t_{2}+\left(1-c_{j}\right) T_{0}^{(j)}\right)\right)$. First we show that our selection of $c_{i}$ and $T_{0}^{(i)}$ guarantees $t_{w} \geq \tau_{j}^{i}\left(t_{2}+W\right)$ due to the following sequence of inequalities:

$$
\begin{aligned}
& 2 a_{\max }^{2} \geq 1, \\
& \Rightarrow \quad 4 W a_{\max }^{2} \geq 2 W, \\
& \Rightarrow \quad\left(\frac{1}{8 n}\right)\left(32 W n a_{\max }^{2}\right) \geq 2 W, \\
& \Rightarrow \quad\left(\frac{i}{5 n-i}\right) T_{0} \geq 2 W, \\
& \Rightarrow \quad c_{i} T_{0}^{(i)} \geq 2 W, \\
& \Rightarrow \quad \frac{c_{i} T_{0}^{(i)}}{a_{i j}} \geq \frac{2 W}{a_{i j}}, \\
& \Rightarrow \quad \frac{c_{i} T_{0}^{(i)}}{a_{i j}} \geq \frac{W}{a_{i j}}+W \\
& \Rightarrow \quad c_{i} T_{0}^{(i)} \geq W+a_{i j} W \text {, } \\
& \Rightarrow \quad \tau_{i}^{j}\left(t_{3}\right)+\frac{c_{i} T_{0}^{(i)}}{a_{i j}} \geq t_{2}+W, \\
& \Rightarrow \quad c_{i} T_{0}^{(i)} \geq t_{3}+W-t_{3}+a_{i j} W, \\
& \Rightarrow \quad c_{i} T_{0}^{(i)} \geq \tau_{j}^{i}\left(t_{2}\right)-t_{3}+a_{i j} W, \\
& \Rightarrow \quad t_{3}+c_{i} T_{0}^{(i)} \geq \tau_{j}^{i}\left(t_{2}\right)+a_{i j} W, \\
& \Rightarrow \quad t_{w} \geq \tau_{j}^{i}\left(t_{2}+W\right) \text {. }
\end{aligned}
$$

Therefore $t_{w} \geq \tau_{j}^{i}\left(t_{2}+W\right)$. Next, we show that our selection of $c_{j}$ and $T_{0}^{(j)}$ guarantees that $t_{w}+W<\tau_{j}^{i}\left(t_{2}+\left(1-c_{i}\right) T_{0}^{(j)}\right)$ due to the following sequence of inequalities:

$$
\begin{array}{ll} 
& 1<8 n a_{\max } \\
\Rightarrow \quad & 2 W a_{\max }<16 W n a_{\max }^{2} \\
\Rightarrow \quad & 2 W a_{\max }<\frac{T_{0}}{2} \\
\Rightarrow & 2 W a_{\max }<\left(1-\frac{2 n a_{\max }}{4 n a_{\max }}\right) T_{0}
\end{array}
$$




$$
\begin{array}{ll}
\Rightarrow & \frac{2 W}{a_{i j}}<\left(1-2 n c_{n} a_{\max }\right) T_{0} \\
\Rightarrow & \frac{2 W}{a_{i j}}<\left(j-\frac{j c_{j}+i c_{i}}{a_{i j}}\right) T_{0} \\
\Rightarrow & \frac{2 W}{a_{i j}}<\left(j\left(1-c_{j}\right)-\frac{i c_{i}}{a_{i j}}\right) T_{0} \\
\Rightarrow & \frac{2 W}{a_{i j}}<j\left(1-c_{j}\right) T_{0}-\frac{i c_{i} T_{0}}{a_{i j}} \\
\Rightarrow & \frac{2 W}{a_{i j}}<\left(1-c_{j}\right) T_{0}^{(j)}-\frac{c_{i} T_{0}^{(i)}}{a_{i j}} \\
\Rightarrow & W+\frac{W}{a_{i j}}<\left(1-c_{j}\right) T_{0}^{(j)}-\frac{c_{i} T_{0}^{(i)}}{a_{i j}} \\
\Rightarrow & a_{i j} W+W<a_{i j}\left(1-c_{j}\right) T_{0}^{(j)}-c_{i} T_{0}^{(i)} \\
\Rightarrow & \tau_{j}^{i}\left(t_{2}\right)+a_{i j} W-\tau_{j}^{i}\left(t_{2}\right)+W<a_{i j}\left(1-c_{j}\right) T_{0}^{(j)}-c_{i} T_{0}^{(i)} \\
\Rightarrow & \tau_{j}^{i}\left(t_{2}+W\right)-\tau_{j}^{i}\left(t_{2}\right)+W<a_{i j}\left(1-c_{j}\right) T_{0}^{(j)}-c_{i} T_{0}^{(i)} \\
\Rightarrow & t_{3}-\tau_{j}^{i}\left(t_{2}\right)+W<a_{i j}\left(1-c_{j}\right) T_{0}^{(j)}-c_{i} T_{0}^{(i)} \\
\Rightarrow & \tau_{i}^{j}\left(t_{3}\right)+\frac{c_{i} T_{0}^{(i)}+W}{a_{i j}}<t_{2}+\left(1-c_{j}\right) T_{0}^{(j)} \\
\Rightarrow & t_{3}+c_{i} T_{0}^{(i)}+W<\tau_{j}^{i}\left(t_{2}\right)+a_{i j}\left(1-c_{j}\right) T_{0}^{(j)} \\
\Rightarrow & t_{w}+W<\tau_{j}^{i}\left(t_{2}+\left(1-c_{j}\right) T_{0}^{(j)}\right) .
\end{array}
$$

It follows from the previous two series of inequalities that $\left[t_{w}, t_{w}+W\right) \subset\left[\tau_{j}^{i}\left(t_{2}+\right.\right.$ $\left.W), \tau_{j}^{i}\left(t_{2}+\left(1-c_{i}\right) T_{0}^{(j)}\right)\right)$. (ii) Now use part (i) to show that node $i$ transmits during the interval $\left[t_{w}, t_{w}+W\right)$ while node $j$ is silent. Let $t \in\left[t_{w}, t_{w}+W\right)$. From the definition of $t_{3}$ and $t_{w}$ we have:

$$
\begin{aligned}
c_{i} T_{0}^{(i)} & =t_{w} \bmod T_{0}^{(i)} \\
& \leq t_{w} \bmod T_{0}^{(i)}+\left(t-t_{w}\right) \bmod T_{0}^{(i)} \\
& <t_{w} \bmod T_{0}^{(i)}+\left(t_{w}+W-t_{w}\right) \bmod T_{0}^{(i)} \\
& =t_{w} \bmod T_{0}^{(i)}+W \bmod T_{0}^{(i)} \\
& =t_{w} \bmod T_{0}^{(i)}+W \\
& =c_{i} T_{0}^{(i)}+W .
\end{aligned}
$$

Therefore $c_{i} T_{0}^{(i)} \leq t_{w} \bmod T_{0}^{(i)}+\left(t-t_{w}\right) \bmod T_{0}^{(i)}<c_{i} T_{0}^{(i)}+W$ for all $\left[t_{w}, t_{w}+W\right)$. It follows from this inequality that:

$$
\begin{aligned}
c_{i} T_{0}^{(i)} & \leq t_{w} \bmod T_{0}^{(i)}+\left(t-t_{w}\right) \bmod T_{0}^{(i)} \\
& =\left[t_{w} \bmod T_{0}^{(i)}+\left(t-t_{w}\right) \bmod T_{0}^{(i)}\right] \bmod T_{0}^{(i)} \\
& =t \bmod T_{0}^{(i)} \\
& <c_{i} T_{0}^{(i)}+W .
\end{aligned}
$$

Therefore $c_{i} T_{0}^{(i)} \leq t \bmod T_{0}^{(i)}<c_{i} T_{0}^{(i)}+W$ for all $t \in\left[t_{w}, t_{w}+W\right)$. It follows from this inequality and the definition of $M_{1}^{(i)}(t)$ that $M_{1}^{(i)}(t)=1$ for all $t \in\left[t_{3}+c_{i} T_{0}^{(i)}, t_{3}+\right.$ $\left.c_{i} T_{0}^{(i)}+W\right)$. So we have shown that node $i$ transmits during the interval $\left[t_{w}, t_{w}+W\right)$. It now remains to show that node $j$ is silent. We have $\tau_{i}^{j}(t) \in\left[t_{2}+W, t_{2}+\left(1-c_{i}\right) T_{0}^{(j)}\right)$ 
since $\tau_{i}^{j}\left(\left[t_{w}, t_{w}+W\right)\right) \subset\left[t_{2}+w, t_{2}+\left(1-c_{j}\right) T_{0}^{(j)}\right)$. It follows from the definition of $t_{2}$ that:

$$
\begin{aligned}
c_{j} T_{0}^{(j)}+W & =\left(t_{2}+W\right) \bmod T_{0}^{(j)} \\
& \leq\left(t_{2}+W\right) \bmod T_{0}^{(j)}+\left(\tau_{i}^{j}(t)-\left(t_{2}+W\right)\right) \bmod T_{0}^{(j)} \\
& <\left(t_{2}+W\right) \bmod T_{0}^{(j)}+\left(t_{2}+\left(1-c_{j}\right) T_{0}^{(j)}-\left(t_{2}+W\right)\right) \bmod T_{0}^{(j)} \\
& =c_{j} T_{0}^{(j)}+W+\left(1-c_{j}\right) T_{0}^{(j)}-W \\
& =T_{0}^{(j)} .
\end{aligned}
$$

Therefore $c_{j} T_{0}^{(j)}+W \leq\left(t_{2}+W\right) \bmod T_{0}^{(j)}+\left(\tau_{i}^{j}(t)-\left(t_{2}+W\right)\right) \bmod T_{0}^{(j)}<T_{0}^{(j)}$ for all $\left[t_{w}, t_{w}+W\right)$. It follows from this fact that:

$$
\begin{aligned}
c_{j} T_{0}^{(j)}+W & \leq\left(t_{2}+W\right) \bmod T_{0}^{(j)}+\left(\tau_{i}^{j}(t)-\left(t_{2}+W\right)\right) \bmod T_{0}^{(j)} \\
& =\left[\left(t_{2}+W\right) \bmod T_{0}^{(j)}+\left(\tau_{i}^{j}(t)-\left(t_{2}+W\right)\right) \bmod T_{0}^{(j)}\right] \bmod T_{0}^{(j)} \\
& =\tau_{i}^{j}(t) \bmod T_{0}^{(j)} \\
& <T_{0}^{(j)} .
\end{aligned}
$$

Therefore $c_{j} T_{0}^{(j)}+W \leq \tau_{i}^{j}(t) \bmod T_{0}^{(j)}<T_{0}^{(j)}$ for all $\left[t_{w}, t_{w}+W\right)$. It follows from this fact and the definition of $M_{1}^{(j)}(t)$ that $M_{1}^{(j)}\left(\tau_{i}^{j}(t)\right)=0$ for all $t \in\left[t_{w}, t_{w}+W\right)$.

Now we prove that node $i$ can successfully transmit a message of length $W$ to node $i$ when $a_{i j} \leq 1$. We show, using the previous three lemmas that within the interval $\left[t_{i}, t_{i}+T_{i}\right)$ there exists a pulse of length $W$ generated by node $i$ that does not collide with a pulse generated by node $j$.

Lemma 3.12. Suppose $a_{i j} \leq 1$ and $i>j$. Given $t_{1}$ such that $\tau_{j}^{i}\left(t_{1}\right) \geq 0$, there exists an interval $\left[t_{w}, t_{w}+W\right)$ that satisfies the following conditions for all $t \in\left[t_{w}, t_{w}+W\right):$

(i) $\left[t_{w}, t_{w}+W\right) \subset\left[t_{1}, t_{1}+T_{n, 2}\right)$,

(ii) $\left[\tau_{i}^{j}\left(t_{w}\right), \tau_{i}^{j}\left(t_{w}+W\right)\right) \subset\left[\tau_{j}^{i}\left(t_{1}\right), \tau_{j}^{i}\left(t_{1}\right)+T_{n, 2}\right)$,

(iii) $M_{1}^{(i)}(t) s_{1}^{(i, j)}(t)=1$,

(iv) $M_{1}^{(j)}\left(\tau_{i}^{j}(t)\right) s_{1}^{(j, i)}\left(\tau_{i}^{j}(t)\right)+s_{1}^{(j, i)}\left(\tau_{i}^{j}(t)\right)=1$.

Proof. It follows from Lemma 3.9 that there exists an interval $\left[t_{i}, t_{i}+T_{i}\right)$ that satisfies the following:

(i) $\left[t_{i}, t_{i}+T_{i}\right) \subset\left[t_{1}, t_{1}+T_{n, 2}\right)$,

(ii) $\left[\tau_{i}^{j}\left(t_{i}\right), \tau_{i}^{j}\left(t_{i}+T_{i}\right)\right) \subset\left[\tau_{i}^{j}\left(t_{1}\right), \tau_{i}^{j}\left(t_{1}\right)+T_{n, 2}\right)$,

(iii) $s_{1}^{(i, j)}(t)=1$,

(iv) $s_{1}^{(j, i)}\left(\tau_{i}^{j}(t)\right)=1$.

Moreover,

$$
\left|\left[t_{i}, t_{i}+T_{i}\right)\right|=T_{i}
$$




$$
\begin{aligned}
& =a_{i j} T_{j-1,2} \\
& \geq a_{i j} T_{0,2} \\
& =a_{i j} 2 T_{n, 1} \\
& =a_{i j} 2\left(\left\lceil n a_{\max }\right\rceil+2\right) T_{0}^{(n)} \\
& \geq 2\left(\frac{\left\lceil n a_{\max }\right\rceil+2}{a_{\max }}\right) T_{0}^{(i)} \\
& \geq 2 n T_{0}^{(i)} .
\end{aligned}
$$

It follows from the inequality $\left|\left[t_{i}, t_{i}+T_{i}\right)\right| \geq 2 n T_{0}^{(i)}$ that there exists an interval $I_{3}:=\left[t_{3}, t_{3}+W\right) \subset\left[t_{i}, t_{i}+T_{i}\right)$ that satisfies the following conditions:

(i) $0 \leq t \bmod T_{0}^{(i)}<W$,

(ii) $0 \leq t_{3}-t_{i}<T_{0}^{(i)}$

There are three cases that may occur:

Case 1: $M_{1}^{(j)}\left(\tau_{i}^{j}(t)\right)=0$, for all $\left[t_{3}, t_{3}+W\right)$. In this case, set $t_{w}:=t_{3}$. It follows from condition (i) that for all $t \in\left[t_{w}, t_{w}+W\right.$ ) we have,

$$
\begin{aligned}
M_{1}^{(j)}\left(\tau_{i}^{j}(t)\right) & =0, \\
M_{1}^{(i)}(t) & =1 .
\end{aligned}
$$

In addition, we clearly have $\left[t_{w}, t_{w}+W\right) \subset\left[t_{i}, t_{i}+T_{i}\right)$.

Case 2: $M_{1}^{(j)}\left(\tau_{i}^{j}(t)\right) \neq 0$ for some $t \in\left[t_{3}, t_{3}+W\right)$. More specifically, there exists an interval $I_{2}:=\left[t_{2}, t_{2}+W\right)$ such that $0 \leq t \bmod T_{0}^{(j)}<W$ for all $t \in I_{2}$ and $\left[t_{2}, t_{2}+W\right) \cap\left[\tau_{i}^{j}\left(t_{3}\right), \tau_{i}^{j}\left(t_{3}+W\right)\right) \neq \emptyset$.

In this case, let $t_{w}:=t_{3}+c_{i} T_{0}^{(i)}$. It follows from Lemma 3.10 that for all $t \in$ $\left[\tau_{i}^{j}\left(t_{w}\right), \tau_{i}^{j}\left(t_{w}+W\right)\right.$ :

$$
\begin{aligned}
M_{1}^{(i)}(t) & =1, \\
M_{1}^{(j)}\left(\tau_{i}^{j}(t)\right) & =0 .
\end{aligned}
$$

Clearly $t_{w} \geq t_{3} \geq t_{i}$. We also can show that $t_{w}+W<t_{i}+T_{i}$ due to the following sequence of inequalities:

$$
\begin{aligned}
t_{w}+W & =t_{3}+c_{i} T_{0}^{(i)}+W \\
& =t_{i}+T_{0}^{(i)}+c_{i} T_{0}^{(i)}+W \\
& <t_{i}+T_{0}^{(i)}+T_{0}^{(i)} \\
& =t_{i}+2 T_{0}^{(i)} \\
& <t_{i}+2 T_{0}^{(n)} \\
& <t_{i}+2 \frac{\left\lceil n a_{\max }\right\rceil+2}{a_{\max }} T_{0}^{(n)}
\end{aligned}
$$




$$
\begin{aligned}
& =t_{i}+\frac{2 T_{n, 1}}{a_{\max }} \\
& =t_{i}+\frac{T_{0,2}}{a_{\max }} \\
& \leq t_{i}+a_{i j} T_{0,2} \\
& \leq t_{i}+a_{i j} T_{j-1,2} \\
& \leq t_{i}+T_{i} .
\end{aligned}
$$

Therefore $\left[t_{w}, t_{w}+W\right) \subset\left[t_{i}, t_{i}+T_{i}\right)$.

Case 3: $M_{1}^{(j)}\left(\tau_{i}^{j}(t)\right) \neq 0$ for all $t \in\left[t_{3}, t_{3}+W\right)$. More specifically, there exists an interval $I_{2}:=\left[t_{2}, t_{2}+W\right)$ such that $c_{j} T_{0}^{(j)} \leq t \bmod T_{0}^{(j)}<c_{j} T_{0}^{(j)}+W$ for all $t \in I_{2}$ and $\left[\tau_{j}^{i}\left(t_{2}\right), \tau_{j}^{i}\left(t_{2}+W\right)\right) \cap\left[t_{3}, t_{3}+W\right) \neq \emptyset$.

In this case let $t_{w}:=t_{3}+c_{i} T_{0}^{(i)}$. It follows from Lemma 3.11 that for all $t \in$ $\left[t_{w}, t_{w}+W\right):$

$$
\begin{aligned}
M_{1}^{(i)}(t) & =1, \\
M_{1}^{(j)}\left(\tau_{i}^{j}(t)\right) & =0 .
\end{aligned}
$$

Clearly $t_{w} \geq t_{3} \geq t_{i}$. We also can show that $t_{w}+W<t_{i}+T_{i}$ due to the following sequence of inequalities:

$$
\begin{aligned}
t_{w}+W & =t_{3}+c_{i} T_{0}^{(i)}+W \\
& \leq t_{i}+T_{0}^{(i)}+c_{i} T_{0}^{(i)}+W \\
& <t_{i}+2 T_{0}^{(i)} \\
& =t_{i}+2 T_{0}^{(n)} \\
& =t_{i}+2 \frac{\left\lceil n a_{\max }\right\rceil+2}{a_{\max }} T_{0}^{(n)} \\
& =t_{i}+\frac{2 T_{n, 1}}{a_{\max }} \\
& =t_{i}+\frac{T_{0,2}}{a_{\max }} \\
& \leq t_{i}+a_{i j} T_{0,2} \\
& \leq t_{i}+a_{i j} T_{j-1,2} \\
& =t_{i}+T_{i} .
\end{aligned}
$$

Therefore $\left[t_{w}, t_{w}+W\right) \subset\left[t_{i}, t_{i}+T_{i}\right)$. It follows that $\left[t_{w}, t_{w}+W\right) \subset\left[t_{i}, t_{i}+T_{i}\right)$ in all 
cases and for all $t \in\left[t_{w}, t_{w}+W\right)$ we have:

$$
\begin{aligned}
M_{1}^{(i)}(t) & =1, \\
M_{1}^{(j)}\left(\tau_{i}^{j}(t)\right) & =0, \\
s_{1}^{(i, j)}(t) & =1, \\
s_{1}^{(j, i)}\left(\tau_{i}^{j}(t)\right) & =1 .
\end{aligned}
$$

Therefore for all $t \in\left[t_{w}, t_{w}+W\right)$ we have:

$$
\begin{aligned}
M_{1}^{(i)}(t) s_{1}^{(i, j)}(t) & =1, \\
M_{1}^{(j)}\left(\tau_{i}^{j}(t)\right) s_{1}^{(j, i)}\left(\tau_{i}^{j}(t)\right)+s_{1}^{(j, i)}\left(\tau_{i}^{j}(t)\right) & =1 .
\end{aligned}
$$

Now we repeat the process when $a_{i j}>1$. In the following lemma we show that for any two pairs of nodes $i$ and $j$, there exists an interval of time, $\left[t_{i}, t_{i}+T_{i}\right)$ with respect to node $i$ 's clock, in which node $i$ and $j$ are scheduled to communicate with each other using the signals $M_{2}^{(i)}(t)$ and $M_{2}^{(j)}(t)$ respectively.

LEMma 3.13. Suppose $i>j$. Given $t_{1}$ such that $\tau_{i}^{j}\left(t_{1}\right) \geq 0$, there exists an interval $\left[t_{i}, t_{i}+T_{i}\right)$ that satisfies the following conditions for all $t \in\left[t_{i}, t_{i}+T_{i}\right)$ :

$$
\begin{aligned}
{\left[t_{i}, t_{i}+T_{i}\right) } & \subset\left[t_{1}, t_{1}+T_{n, 2}\right), \\
{\left[\tau_{i}^{j}\left(t_{i}\right), \tau_{i}^{j}\left(t_{i}+T_{i}\right)\right) } & \subset\left[\tau_{i}^{j}\left(t_{1}\right), \tau_{i}^{j}\left(t_{1}\right)+T_{n, 2}\right), \\
s_{2}^{(j, i)}\left(\tau_{i}^{j}(t)\right) & =1, \\
s_{2}^{(i, j)}(t) & =1 .
\end{aligned}
$$

Proof. The proof of this lemma is very similar to the proof of Lemma 3.9.

First, we consider case one in which a primary pulse generated by node $i$ collides with a primary pulse generated by node $j$. We show that there exists a pulse of length $W$ generated by node $i$ that does not collide with node $i$.

Lemma 3.14. Assume $i>j$ and $a_{i j}>1$. Let $I_{3}:=\left[t_{3}, t_{3}+W\right)$ be a primary pulse of $M_{2}^{(i)}(t)$ with respect to node $i$ 's clock and let $I_{2}:=\left[t_{2}, t_{2}+W\right)$ be a primary pulse of $M_{2}^{(j)}(t)$ with respect to node $j$ 's clock. Suppose the two pulses overlap. That is, $\left[t_{3}, t_{3}+W\right) \cap \tau_{j}^{i}\left(\left[t_{2}, t_{2}+W\right)\right) \neq \emptyset$. Then there exists a pulse at $t_{w}:=t_{3}+k T_{0}^{(n-i+1)}$ with respect to node $i$ 's clock, where $k=\left\lfloor\frac{1}{a_{j i}}\left(\frac{n-j+1}{n-i+1}\right)-\frac{W}{T_{0}^{(n-i+1)}}-\frac{W}{a_{j i} T_{0}^{(n-i+1)}}-c_{n-i+1}\right\rfloor$, that does not overlap with any pulse of $M_{2}^{(j)}\left(\tau_{i}^{j}(t)\right)$.

Proof. Let $\tilde{i}:=n-i+1$ and $\tilde{j}:=n-j+1$. Then $I_{3}$ is a primary pulse of $M_{1}^{(\tilde{i})}(t)$ and $I_{2}$ is a primary pulse of $M_{1}^{(\tilde{j})}(t)$. Set $a_{\tilde{i} \tilde{j}}:=a_{i j}$. Therefore $a_{\tilde{j} \tilde{i}} \leq 1$. We need to show that there exists a pulse at $t_{w}:=t_{3}+k T_{0}^{(\tilde{i})}$ with respect to node $\tilde{i}$ 's clock 
that does not overlap with any pulse of $M_{1}^{(\tilde{j})}\left(\tau_{\tilde{i}}^{\tilde{j}}(t)\right)$. This result was already shown in Lemma 3.2.

Next we consider case two in which a primary pulse generated by node $i$ collides with a secondary pulse generated by node $j$. We show that there exists a pulse of length $W$ generated by node $i$ that does not collide with node $j$.

Lemma 3.15. Assume $i>j$ and $a_{i j}>1$. Let $I_{3}:=\left[t_{3}, t_{3}+W\right)$ be a secondary pulse of $M_{2}^{(i)}(t)$ with respect to node $i$ 's clock and let $I_{2}:=\left[t_{2}, t_{2}+W\right)$ be a primary pulse of $M_{2}^{(j)}(t)$ with respect to node j's clock. Suppose the two pulses overlap. That is, $\left[t_{2}, t_{2}+W\right) \cap \tau_{i}^{j}\left(\left[t_{3}, t_{3}+W\right)\right) \neq \emptyset$. Then there exists a pulse at $t_{w}:=t_{3}+c_{n-i+1} T_{0}^{(n-i+1)}$ with respect to node $i$ 's clock that does not overlap with any pulse of $M_{2}^{(j)}\left(\tau_{i}^{j}(t)\right)$.

Proof. Let $\tilde{i}:=n-i+1$ and $\tilde{j}:=n-j+1$. Then $I_{3}$ is a primary pulse of $M_{1}^{(\tilde{i})}(t)$ and $I_{2}$ is a primary pulse of $M_{1}^{(\tilde{j})}(t)$. Set $a_{\tilde{i} \tilde{j}}:=a_{i j}$. Therefore $a_{\tilde{j} \tilde{i}} \leq 1$. We need to show that there exists a pulse at $t_{w}:=t_{3}+c_{\tilde{i}} T_{0}^{(\tilde{i})}$ with respect to node $\tilde{i}^{\prime}$ 's clock that does not overlap with any pulse of $M_{1}^{(\tilde{j})}\left(\tau_{\tilde{i}}^{\tilde{j}}(t)\right)$. This result was already shown in Lemma 3.3.

Now we prove that node $i$ can successfully transmit a message of length $W$ to node $i$ when $a_{i j}<1$. We show, using the previous three lemmas, that within the interval $\left[t_{i}, t_{i}+T_{i}\right)$ there exists a pulse of length $W$ generated by node $i$ that does not collide with a pulse generated by node $j$.

Lemma 3.16. Suppose $a_{i j}>1$ and $i>j$. Given $t_{1}$ such that $\tau_{i}^{j}\left(t_{1}\right) \geq 0$, there exists an interval $\left[t_{w}, t_{w}+W\right)$ that satisfies the following conditions for all $t \in\left[t_{w}, t_{w}+W\right):$

(i) $\left[t_{w}, t_{w}+W\right) \subset\left[t_{1}, t_{1}+T_{n, 2}\right)$,

(ii) $\left[\tau_{i}^{j}\left(t_{w}\right), \tau_{i}^{j}\left(t_{w}+T_{n, 2}\right)\right) \subset\left[\tau_{i}^{j}\left(t_{1}\right)+T_{n, 2}\right)$,

(iii) $M_{2}^{(i)}(t) s_{2}^{(i, j)}(t)=1$,

(iv) $M_{2}^{(j)}\left(\tau_{i}^{j}(t)\right) s_{2}^{(j, i)}\left(\tau_{i}^{j}(t)\right)+s_{2}^{(j, i)}\left(\tau_{i}^{j}(t)\right)=1$.

We have proved that node $i$ can communicate with node $j$ if $a_{i j} \leq 1$ and if $a_{i j}>1$. Putting both cases together gives us our second theorem; the orthogonal MAC code enables node $i$ to successfully transmit a message of length $W$ units to node $j$ within a finite time $T_{M A C}(W):=T_{n, 2}$ as long as the relative skew $a_{i j} \leq a_{\max }$.

TheOREm 3.2. Without loss of generality, assume $i>j$. Assume there exists $t_{1}$ such that $\tau_{i}^{j}\left(t_{1}\right)>0$. There exists an interval $\left[t_{w}, t_{w}+W\right)$ that satisfies the following conditions for all $t \in\left[t_{w}, t_{w}+W\right)$ and some $k \in\{1,2\}$ :

(i) $\left[t_{w}, t_{w}+W\right) \subset\left[t_{1}, t_{1}+T_{n, 2}\right)$

(ii) $\left[\tau_{i}^{j}\left(t_{w}\right), \tau_{i}^{j}\left(t_{w}+W\right)\right) \subset\left[\tau_{i}^{j}\left(t_{1}\right), \tau_{i}^{j}\left(t_{1}\right)+T_{n, 2}\right)$

(iii) $M_{k}^{(i)}(t) s_{k}^{(i, j)}(t)=1$

(iv) $M_{k}^{(j)}\left(\tau_{i}^{j}(t)\right) s_{k}^{(j, i)}\left(\tau_{i}^{j}(t)\right)+s_{k}^{(j, i)}\left(\tau_{i}^{j}(t)\right)=1$.

Proof. There are two cases to consider: 
Case 1: $a_{i j} \leq 1$. It follows from Lemma 3.12 that there exists an interval $\left[t_{w}, t_{w}+W\right)$ that satisfies the following conditions for all $t \in\left[t_{w}, t_{w}+W\right)$ :

(i) $\left[t_{w}, t_{w}+W\right) \subset\left[t_{1}, t_{1}+T_{n, 2}\right)$,

(ii) $\left[\tau_{i}^{j}\left(t_{w}\right), \tau_{i}^{j}\left(t_{w}+W\right)\right) \subset\left[\tau_{i}^{j}\left(t_{1}\right), \tau_{i}^{j}\left(t_{1}\right)+T_{n, 2}\right)$,

(iii) $M_{1}^{(i)}(t) s_{1}^{(i, j)}(t)=1$

(iv) $M_{1}^{(j)}\left(\tau_{i}^{j}(t)\right) s_{1}^{(j, i)}\left(\tau_{i}^{j}(t)\right)+s_{1}^{(j, i)}\left(\tau_{i}^{j}(t)\right)=1$.

Case 2: $a_{i j}>1$. It follows from Lemma 3.16 that there exists an interval $\left[t_{w}, t_{w}+W\right)$ that satisfies the following conditions for all $t \in\left[t_{w}, t_{w}+W\right)$ :

(i) $\left[t_{w}, t_{w}+W\right) \subset\left[t_{1}, t_{1}+T_{n, 2}\right)$,

(ii) $\left[\tau_{i}^{j}\left(t_{w}\right), \tau_{i}^{j}\left(t_{w}+W\right)\right) \subset\left[\tau_{i}^{j}\left(t_{1}\right), \tau_{i}^{j}\left(t_{1}\right)+T_{n, 2}\right)$,

(iii) $M_{2}^{(i)}(t) s_{2}^{(i, j)}(t)=1$,

(iv) $M_{2}^{(j)}\left(\tau_{i}^{j}(t)\right) s_{2}^{(j, i)}\left(\tau_{i}^{j}(t)\right)+s_{2}^{(j, i)}\left(\tau_{i}^{j}(t)\right)=1$.

The proof of Theorem 2.1 follows from Theorem 3.2 and Theorem 3.1.

4. Analysis of the Orthogonal MAC code Complexity. In this section we analyze the length of the orthogonal MAC code with respect to the size of the message $W$, the number of nodes $n$, and the maximum relative skew $a_{\max }$. The following lemma determines the length of the orthogonal MAC code for message of size $W$.

LEMMA 4.1. The length of the orthogonal MAC code for a message of size $W$ is at most $T_{M A C}(W)$, where $T_{M A C}(W):=\left(2 n a_{\max }\right)^{9 n} W$ and the length is measured with respect to the local clock.

Proof. The length of the orthogonal MAC code is $T_{n, 2}$ where $T_{n, 2}$ is recursively defined in (5) by:

$$
T_{i, 2}:=2 a_{\max } n T_{i-1,2},
$$

where by (4),

$$
T_{0,2}:=2\left(\left\lceil n a_{\max }\right\rceil+2\right) n\left(32 W n\left(a_{\max }\right)^{2}\right) .
$$

It follows that for all $i \leq n$ :

$$
\begin{aligned}
T_{i, 2} & :=\left(2 a_{\max } n\right)^{i} T_{0,2} \\
& =\left(2 a_{\max } n\right)^{i} 2\left(\left\lceil n a_{\max }\right\rceil+2\right) n\left(32 W n\left(a_{\max }\right)^{2}\right) \\
& \leq\left(2 a_{\max } n\right)^{i} 2\left(3 n a_{\max }\right) n\left(32 W n\left(a_{\max }\right)^{2}\right) \\
& \leq 192\left(2 a_{\max } n\right)^{i}\left(n a_{\max }\right)^{3} W \\
& \leq\left(2 n a_{\max }\right)^{8}\left(2 n a_{\max }\right)^{i} W \\
& \leq\left(2 n a_{\max }\right)^{i+8} W \\
& \leq\left(2 n a_{\max }\right)^{9 n} W .
\end{aligned}
$$


Therefore $T_{n, 2} \leq\left(2 n a_{\max }\right)^{9 n} W$ and the lemma is proved.

The length of the orthogonal MAC code is doubly exponential in the number of nodes $n$; a property that makes this code infeasible to implement for large values of $n$. However, in the context of the larger protocol suite described in the introduction, the overhead of the orthogonal MAC code can be mitigated by choosing a data transfer phase on a much larger time-scale. Hence the orthogonal MAC code plays a critical role in obtaining a theoretical result; the existance of a protocol suite that enables a network of asynchronous nodes to form a fully functioning network operating at near optimal utility.

In practice, we cannot rely on arbitrarily large data data transfer phases to hide the overhead of the orthogonal MAC code, so it is worth examining ways to reduce the code complexity. The construction of $s_{k}^{(i, j)}(t)$, which determines the intervals in which node $i$ corresponds with node $j$, is designed to guarantee that an interval in which $s_{k}^{(i, j)}(t)=1$ overlaps with an interval in which $s_{k}^{(j, i)}\left(\tau_{i}^{j}(t)\right)=1$ for all node pairs $i$ and $j$. In this paper, we adopted the obvious approach and made the pulse of $s_{k}^{(i, j)}(t)$ more than $n a_{\max }$ times larger than the pulse of $s_{k}^{(j, i)}(t)$, where $i>j$. This approach however, leads to the recursive definition of $T_{i, 2}$ that is ultimately doubly exponential in $n$. Any reduction in the code complexity requires a new and less obvious design of $s_{k}^{(i, j)}(t)$.

5. Conclusions and future work. The orthogonal MAC code presented in this paper allows a collection of half-duplex, unsynchronized nodes with affine clocks not ticking at the same rate and unaware of each others rates and offsets to exchange messages of size $W$ units within a bounded time $T_{M A C}(W)$. This code is part a larger protocol suite that enables the collection to form a fully functioning network even while under sustained attack by hidden malicious nodes. Although this paper focuses on proving the existence a MAC code with the desired capability, it is likely that other important factors such as complexity and efficiency can also be improved. For example, currently the length of the orthogonal MAC code is proportional to $n^{n}$, where $n$ is the number of nodes. The double exponential originates from the part of the protocol that guarantees an interval in which any pair of nodes pay attention to each other. However, the approach taken was admittedly brute-force and can probably be significantly refined. It would be interesting to determine whether there are any fundamental limits to the efficiency of the MAC code and whether these limits are achievable.

\section{REFERENCES}

[1] Norman Abramson, The ALOHA system: another alternative for computer communications. In: Proceedings of the November 17-19, 1970, Fall Joint Computer Conference, AFIPS '70 
(Fall), pages 281-285, New York, NY, USA, 1970. ACM.

[2] R. Gold, Optimal binary sequences for spread spectrum multiplexing (corresp.). Information Theory, IEEE Transactions on, 13:4(1967), pp. 619-621.

[3] Y-C. Hu, J. Ponniah, and P. R. Kumar, An Axiomatic Clean Slate Approach to Secure Wireless Networking. MOBIHOC, submitted for publication 2012. 
